\title{
NILPOTENT ORBITS IN CLASSICAL LIE ALGEBRAS OVER FINITE FIELDS OF CHARACTERISTIC 2 AND THE SPRINGER CORRESPONDENCE
}

\author{
TING XUE
}

\begin{abstract}
Let $G$ be an adjoint algebraic group of type $B, C$, or $D$ over an algebraically closed field of characteristic 2 . We construct a Springer correspondence for the Lie algebra of $G$. In particular, for orthogonal Lie algebras in characteristic 2 , the structure of component groups of nilpotent centralizers is determined and the number of nilpotent orbits over finite fields is obtained.
\end{abstract}

\section{INTRODUCTION}

Throughout this paper, $\mathbf{k}$ denotes an algebraically closed field of characteristic $2, \mathbf{F}_{q}$ denotes a finite field of characteristic 2 and $\overline{\mathbf{F}}_{q}$ denotes an algebraic closure of $\mathbf{F}_{q}$.

In 2, Hesselink determines the nilpotent orbits in classical Lie algebras under the adjoint action of classical Lie groups over k. In [9], Spaltenstein gives a parametrization of these nilpotent orbits by pairs of partitions. We extend Hesselink's method to study the nilpotent orbits in the Lie algebras of orthogonal groups over $\mathbf{F}_{q}$. Using this extension and Spaltenstein's parametrization we classify the nilpotent orbits over $\mathbf{F}_{q}$. We determine the structure of the component groups of centralizers of nilpotent elements. In particular, we obtain the number of nilpotent orbits over $\mathbf{F}_{q}$.

Let $G$ be a connected reductive algebraic group over an algebraically closed field and $\mathfrak{g}$ the Lie algebra of $G$. When the characteristic of the field is large enough, Springer [12] constructs representations of the Weyl group of $G$ which are related to the nilpotent $G$-orbits in $\mathfrak{g}$. Lusztig 4 constructs the generalized Springer correspondence in all characteristics which are related to the unipotent conjugacy classes in $G$. Let $G_{a d}$ be an adjoint algebraic group of type $B, C$ or $D$ over k and $\mathfrak{g}_{a d}$ the Lie algebra of $G_{a d}$. We use a similar construction as in [4, [5] to give the Springer correspondence for $\mathfrak{g}_{a d}$. Let $\mathfrak{A}_{a d}$ be the set of all pairs $(\mathrm{c}, \mathcal{F})$ where c is a nilpotent $G_{a d}$-orbit in $\mathfrak{g}_{a d}$ and $\mathcal{F}$ is an irreducible $G_{a d}$-equivariant local system on c (up to isomorphism). We construct a bijective map from the set of isomorphism classes of irreducible representations of the Weyl group of $G_{a d}$ to the set $\mathfrak{A}_{a d}$. In the case of a symplectic group a Springer correspondence (with a different definition than ours) has been established in [3]; in that case centralizers of the nilpotent elements are connected [9]. A complicating feature in the orthogonal case is the existence of non-trivial equivariant local systems on a nilpotent orbit.

Received by the editors December 31, 2008 and, in revised form, June 27, 2009.

2000 Mathematics Subject Classification. Primary 14L35; Secondary 17B10.

(C)2009 American Mathematical Society Reverts to public domain 28 years from publication 
Most of the results in this paper have been announced in [16].

\section{HESSELINK'S CLASSIFICATION OF NILPOTENT ORBITS OVER AN ALGEBRAICALLY CLOSED FIELD}

We recall the results of Hesselink about nilpotent orbits in orthogonal algebras in this section (see 2]). Let $\mathbb{K}$ be a field of characteristic 2 , not necessarily algebraically closed.

2.1. A form space $V$ is a finite dimensional vector space over $\mathbb{K}$ equipped with a quadratic form $Q: V \rightarrow \mathbb{K}$. Let $\langle\cdot, \cdot\rangle: V \times V \rightarrow \mathbb{K}$ be the bilinear form $\langle v, w\rangle=Q(v+w)+Q(v)+Q(w)$. Let $V^{\perp}=\{v \in V \mid\langle v, w\rangle=0, \forall w \in V\}$. A form space $V$ is called non-defective if $V^{\perp}=\{0\}$, otherwise, it is called defective. $V$ is called non-degenerate if $\operatorname{dim}\left(V^{\perp}\right) \leq 1$ and $Q(v) \neq 0$ for all non-zero $v \in V^{\perp}$.

Let $V$ be a non-degenerate form space of dimension $N$ over $\mathbb{K}$. Define the orthogonal group $O(V)$ to be $\{g \in \mathrm{GL}(V) \mid Q(g v)=Q(v), \forall v \in V\}$ and define $\mathfrak{o}(V)$ to be $\{x \in \operatorname{End}(V) \mid\langle x v, v\rangle=0, \forall v \in V$ and $\operatorname{tr}(x)=0\}$. In the case where $\mathbb{K}$ is algebraically closed, let $S O(V)$ be the identity component of $O(V)$ and we define $O_{N}(\mathbb{K}), \mathfrak{o}_{N}(\mathbb{K})$ and $S O_{N}(\mathbb{K})$ to be $O(V), \mathfrak{o}(V)$ and $S O(V)$, respectively.

2.2. A form module is defined to be a pair $(V, T)$ where $V$ is a non-degenerate form space and $T$ is a nilpotent element in $\mathfrak{o}(V)$. Classifying nilpotent orbits in $\mathfrak{o}(V)$ is equivalent to classifying form modules $(V, T)$ on the form space $V$. Let $A=\mathbb{K}[[t]]$ be the ring of formal power series in the indeterminate $t$. The form module $V=(V, T)$ is considered as an $A$-module by $\left(\sum_{n \geq 0} a_{n} t^{n}\right) v=\sum_{n \geq 0} a_{n} T^{n} v$.

Let $E$ be the vector space spanned by the linear functionals $t^{-n}: A \rightarrow \mathbb{K}, \sum a_{i} t^{i}$ $\mapsto a_{n}, n \geq 0$. Let $E_{0}$ be the subspace $\sum_{n \geq 0} \mathbb{K} t^{-2 n}$ and $\pi_{0}: E \rightarrow E_{0}$ the natural projection. The space $E$ is considered as an $A$-module by $(a u)(b)=u(a b)$, for $a, b \in A, u \in E$.

An abstract form module is defined to be an $A$-module $V$ with $\operatorname{dim}(V)<\infty$, which is equipped with mappings $\varphi: V \times V \rightarrow E$ and $\psi: V \rightarrow E_{0}$ satisfying the following axioms:

(i) The map $\varphi(\cdot, w)$ is $A$-linear for every $w \in V$.

(ii) $\varphi(v, w)=\varphi(w, v)$ for all $v, w \in V$.

(iii) $\varphi(v, v)=0$ for all $v \in V$.

(iv) $\psi(v+w)=\psi(v)+\psi(w)+\pi_{0}(\varphi(v, w))$ for all $v, w \in V$.

(v) $\psi(a v)=a^{2} \psi(v)$ for $v \in V, a \in A$.

The following proposition identifies a form module $(V, T)$ and the corresponding abstract form module $V=(V, \varphi, \psi)$.

Proposition $2.1([2])$. If $(V, \varphi, \psi)$ is an abstract form module, then $(V,\langle\cdot, \cdot\rangle, Q)$ given by (i) is a form module. If $(V,\langle\cdot, \cdot\rangle, Q)$ is a form module, there is a unique abstract form module $(V, \varphi, \psi)$ such that (i) holds; it is given by (ii).

(i) $\langle v, w\rangle=\varphi(v, w)(1), Q(v)=\psi(v)(1)$.

(ii) $\varphi(v, w)=\sum_{n \geq 0}\left\langle t^{n} v, w\right\rangle t^{-n}, \psi(v)=\sum_{n \geq 0} Q\left(t^{n} v\right) t^{-2 n}$. 
2.3. An element in $\mathfrak{o}(V)$ is nilpotent if and only if it is nilpotent in $\operatorname{End}(V)$. Let $T$ be a nilpotent element in $\mathfrak{o}(V)$. There exists a unique sequence of integers $p_{1} \geq \cdots \geq$ $p_{s} \geq 1$ and a family of vectors $v_{1}, \ldots, v_{s}$ such that $T^{p_{i}} v_{i}=0$ and the vectors $T^{q_{i}} v_{i}$, $0 \leq q_{i} \leq p_{i}-1$ form a basis of $V$. We write $p(V, T)=\left(p_{1}, \ldots, p_{s}\right)$. Define the index function $\chi(V, T): \mathbb{N} \rightarrow \mathbb{Z}$ by $\chi(V, T)(m)=\min \left\{k \geq 0 \mid T^{m} v=0 \Rightarrow Q\left(T^{k} v\right)=0\right\}$. Define $\mu(V)$ to be the minimal integer $m \geq 0$ such that $t^{m} V=0$. For $v \in V$ (or $E)$, we define $\mu(v)=\min \left\{m \geq 0 \mid t^{m} v=0\right\}$.

2.4. Let $V$ be a form module. An orthogonal decomposition of $V$ is an expression of $V$ as a direct sum $V=\sum_{i=1}^{r} V_{i}$ of mutually orthogonal submodules $V_{i}$. The form module $V$ is called indecomposable if $V \neq 0$ and for every orthogonal decomposition $V=V_{1} \oplus V_{2}$ we have $V_{1}=0$ or $V_{2}=0$. Every form module $V$ has some orthogonal decomposition $V=\sum_{i=1}^{r} V_{i}$ in indecomposable submodules $V_{1}, V_{2}, \ldots, V_{r}$. The indecomposable modules are classified as follows.

Proposition 2.2 ([2]). Let $V$ be a non-degenerate indecomposable form module. There exist $v_{1}, v_{2} \in V$ such that $V=A v_{1} \oplus A v_{2}$ and $\mu\left(v_{1}\right) \geq \mu\left(v_{2}\right)$. For any such pair we put $m=\mu\left(v_{1}\right), m^{\prime}=\mu\left(v_{2}\right), \Phi=\varphi\left(v_{1}, v_{2}\right)$ and $\Psi_{i}=\psi\left(v_{i}\right)$. One of the following conditions holds:

(i) $m^{\prime}=\mu(\Phi)=m, \mu\left(\Psi_{i}\right) \leq 2 m-1$.

(ii) $m^{\prime}=\mu(\Phi)=m-1, \mu\left(\Psi_{1}\right)=2 m-1>\mu\left(\Psi_{2}\right)$.

Conversely, let $m \in \mathbb{N}, m^{\prime} \in \mathbb{N} \cup\{0\}, \Phi \in E, \Psi_{1}, \Psi_{2} \in E_{0}$ be given satisfying (i) or (ii). Up to a canonical isomorphism there exists a unique form module $V=$ $A v_{1} \oplus A v_{2}$ with $m=\mu\left(v_{1}\right), m^{\prime}=\mu\left(v_{2}\right), \Phi=\varphi\left(v_{1}, v_{2}\right)$ and $\Psi_{i}=\psi\left(v_{i}\right)$. This form module is indecomposable. In case (i) it is non-defective. In case (ii) it is defective and non-degenerate.

From now on assume $\mathbb{K}$ is algebraically closed. The indecomposable modules in Proposition 2.2 are normalized in [2], Sections 3.4 and 3.5 as follows.

Proposition $2.3([2])$. The indecomposable non-degenerate form modules over $\mathbb{K}$ are:

(i) $W_{l}(m)=A v_{1} \oplus A v_{2},\left[\frac{m+1}{2}\right] \leq l \leq m, \mu\left(v_{1}\right)=\mu\left(v_{2}\right)=m, \psi\left(v_{1}\right)=t^{2-2 l}$, $\psi\left(v_{2}\right)=0$ and $\varphi\left(v_{1}, v_{2}\right)=t^{1-m}([\alpha]$ means the integer part of $\alpha)$.

(ii) $D(m)=A v_{1} \oplus A v_{2}, \mu\left(v_{1}\right)=m, \mu\left(v_{2}\right)=m-1, \psi\left(v_{1}\right)=t^{2-2 m}, \psi\left(v_{2}\right)=0$ and $\varphi\left(v_{1}, v_{2}\right)=t^{2-m}$.

We have $\chi_{W_{l}(m)}=[m ; l]$ and $\chi_{D(m)}=[m ; m]$, where $[m ; l]: \mathbb{N} \rightarrow \mathbb{Z}$ is defined by $[m ; l](n)=\max \{0, \min \{n-m+l, l\}\}$. Among these types of indecomposable modules only the types $D(m)$ are defective.

Remark 2.4. The notation we use here is slightly different from that of 2 . The form module $W_{\left[\frac{m+1}{2}\right]}(m)$ in (i) is isomorphic to the form module $W(m)$ in [2].

Finally, this normalization of indecomposable modules is used to classify all the form modules. Let $(V, T)$ be a non-degenerate from module with $p(V, T)=$ $\left(\lambda_{1}, \ldots, \lambda_{1}, \ldots, \lambda_{k}, \ldots, \lambda_{k}\right)$ where $\lambda_{1}>\cdots>\lambda_{k} \geq 1$ and index function $\chi=$ $\chi(V, T)$. Let $m_{i} \in \mathbb{N}$ be the multiplicity of $\lambda_{i}$ in $p(V, T)$. The isomorphism class of $(V, T)$ is determined by the symbol

$$
S(V, T)=\left(\lambda_{1}\right)_{\chi\left(\lambda_{1}\right)}^{m_{1}}\left(\lambda_{2}\right)_{\chi\left(\lambda_{2}\right)}^{m_{2}} \cdots\left(\lambda_{k}\right)_{\chi\left(\lambda_{k}\right)}^{m_{k}} .
$$


A symbol $S$ of the above form is the symbol of an isomorphism class of nondegenerate form modules if and only if it satisfies the following conditions:

(i) $\chi\left(\lambda_{i}\right) \geq \chi\left(\lambda_{i+1}\right)$ and $\lambda_{i}-\chi\left(\lambda_{i}\right) \geq \lambda_{i+1}-\chi\left(\lambda_{i+1}\right)$, for $i=1, \ldots, k-1$;

(ii) $\frac{\lambda_{i}}{2} \leq \chi\left(\lambda_{i}\right) \leq \lambda_{i}$, for $i=1, \ldots, k$;

(iii) $\chi\left(\lambda_{i}\right)=\lambda_{i}$ if $m_{i}$ is odd, for $i=1, \ldots, k$;

(iv) $\left\{\lambda_{i} \mid m_{i}\right.$ odd $\}=\{m, m-1\} \cap \mathbb{N}$ for some $m \in \mathbb{Z}$.

In the following we denote by a symbol either a form module in the isomorphism class or the corresponding nilpotent orbit.

\section{Indecomposable modules over $\mathbf{F}_{q}$}

In this section, we study the non-degenerate indecomposable form modules over $\mathbf{F}_{q}$. Note that the classification of the indecomposable modules (Proposition 2.2) is valid over any field. Similar to 2, Section 3.5, the non-degenerate indecomposable form modules over $\mathbf{F}_{q}$ are normalized as follows. Fix an element $\delta$ in $\mathbf{F}_{q}$ such that $\delta \notin\left\{x^{2}+x \mid x \in \mathbf{F}_{q}\right\}$.

Proposition 3.1. The non-degenerate indecomposable form modules over $\boldsymbol{F}_{q}$ are:

(i) $W_{l}^{0}(m)=A v_{1} \oplus A v_{2},\left[\frac{m+1}{2}\right] \leq l \leq m$, with $\mu\left(v_{1}\right)=\mu\left(v_{2}\right)=m, \psi\left(v_{1}\right)=$ $t^{2-2 l}, \psi\left(v_{2}\right)=0$ and $\varphi\left(v_{1}, v_{2}\right)=t^{1-m}$;

(ii) $W_{l}^{\delta}(m)=A v_{1} \oplus A v_{2}, \frac{m+1}{2} \leq l \leq m$, with $\mu\left(v_{1}\right)=\mu\left(v_{2}\right)=m, \psi\left(v_{1}\right)=t^{2-2 l}$, $\psi\left(v_{2}\right)=\delta t^{2 l-2 m}$ and $\varphi\left(v_{1}, v_{2}\right)=t^{1-m}$;

(iii) $D(m)=A v_{1} \oplus A v_{2}$ with $\mu\left(v_{1}\right)=m, \mu\left(v_{2}\right)=m-1, \psi\left(v_{1}\right)=t^{2-2 m}, \psi\left(v_{2}\right)=0$ and $\varphi\left(v_{1}, v_{2}\right)=t^{2-m}$.

We have $\chi_{W_{l}^{0}(m)}=\chi_{W_{l}^{\delta}(m)}=[m ; l]$ and $\chi_{D(m)}=[m ; m]$. Among these types only the types $D(m)$ are defective.

Proof. As pointed out in [2], the form modules in Proposition 2.2(ii) over $\mathbf{F}_{q}$ can be normalized the same as in Proposition 2.3(ii). Namely, there exist $v_{1}$ and $v_{2}$ such that the above modules have the form (iii).

Now let $U(m)=A v_{1} \oplus A v_{2}$ be a form module as in Proposition 2.2(i) with $\mu\left(v_{1}\right)=\mu\left(v_{2}\right)=m$, we can assume $\mu\left(\Psi_{1}\right) \geq \mu\left(\Psi_{2}\right)$. We have the following cases:

Case 1: $\Psi_{1}=\Psi_{2}=0$. We can assume $\Phi=t^{1-m}$. Let $\tilde{v}_{1}=v_{1}+t^{m+1-2\left[\frac{m+1}{2}\right]} v_{2}$ and $\tilde{v}_{2}=v_{2}$. We have $\psi\left(\tilde{v}_{1}\right)=t^{2-2\left[\frac{m+1}{2}\right]}, \psi\left(\tilde{v}_{2}\right)=0, \varphi\left(\tilde{v}_{1}, \tilde{v}_{2}\right)=t^{1-m}$.

Case 2: $\Psi_{1} \neq 0, \Psi_{2}=0$. We can assume $\Phi=t^{1-m}$ and $\Psi_{1}=t^{-2 l}$, where $l \leq m-1$. If $l<\left[\frac{m-1}{2}\right]$, let $\tilde{v}_{1}=v_{1}+t^{m+1-2\left[\frac{m+1}{2}\right]} v_{2}+t^{m-2 l-1} v_{2}, \tilde{v}_{2}=v_{2}$; otherwise, let $\tilde{v}_{1}=v_{1}, \tilde{v}_{2}=v_{2}$. Then we get $\psi\left(\tilde{v}_{1}\right)=t^{-2 l},\left[\frac{m-1}{2}\right] \leq l \leq m-1, \psi\left(\tilde{v}_{2}\right)=$ $0, \varphi\left(\tilde{v}_{1}, \tilde{v}_{2}\right)=t^{1-m}$.

Case 3: $\Psi_{1} \neq 0, \Psi_{2} \neq 0$. We can assume $\Psi_{1}=t^{-2 l_{1}}, \Phi=t^{1-m}, \Psi_{2}=$ $\sum_{i=0}^{l_{2}} a_{i} t^{-2 i}$ with $l_{2} \leq l_{1} \leq m-1$.

(1) $l_{1}<\left[\frac{m}{2}\right]$, let $\tilde{v}_{2}=v_{2}+\sum_{i=0}^{m-1} x_{i} t^{i} v_{1}$, then $\psi\left(\tilde{v}_{2}\right)=0$ has a solution for all $x_{i}$ 's and we get Case 2 .

(2) $l_{1} \geq\left[\frac{m}{2}\right]$, let $\tilde{v}_{2}=v_{2}+\sum_{i=0}^{m-1} x_{i} t^{i} v_{1}$. If $a_{m-l_{1}-1} \in\left\{x^{2}+x \mid x \in \mathbf{F}_{q}\right\}$, then $\psi\left(\tilde{v}_{2}\right)=0$ has a solution for all $x_{i}$ 's and we get Case 2. If $a_{m-l_{1}-1} \notin$ $\left\{x^{2}+x \mid x \in \mathbf{F}_{q}\right\}$, then $\psi\left(\tilde{v}_{2}\right)=\delta t^{-2\left(m-l_{1}-1\right)}$ has a solution for all $x_{i}$ 's.

Summarizing Cases 1-3, we have normalized $U(m)=A v_{1} \oplus A v_{2}$ with $\mu\left(v_{1}\right)=$ $\mu\left(v_{2}\right)=m$ as follows:

(i) $\left[\frac{m+1}{2}\right] \leq \chi(m)=l \leq m, \psi\left(v_{1}\right)=t^{2-2 l}, \psi\left(v_{2}\right)=0, \varphi\left(v_{1}, v_{2}\right)=t^{1-m}$, denoted by $W_{l}^{0}(m)$. 
(ii) $\frac{m+1}{2} \leq \chi(m)=l \leq m, \psi\left(v_{1}\right)=t^{2-2 l}, \psi\left(v_{2}\right)=\delta t^{-2(m-l)}, \varphi\left(v_{1}, v_{2}\right)=t^{1-m}$, denoted by $W_{l}^{\delta}(m)$.

One can verify that these form modules are not isomorphic to each other.

Remark 3.2. It follows that the isomorphism class of the form module $W_{l}(m)$ over $\overline{\mathbf{F}}_{q}$ remains as one isomorphism class over $\mathbf{F}_{q}$ when $l=\frac{m}{2}$ and decomposes into two isomorphism classes $W_{l}^{0}(m)$ and $W_{l}^{\delta}(m)$ over $\mathbf{F}_{q}$ when $l \neq \frac{m}{2}$. The isomorphism class of the form module $D(m)$ over $\overline{\mathbf{F}}_{q}$ remains as one isomorphism class over $\mathbf{F}_{q}$.

\section{Nilpotent orbits OVer $\mathbf{F}_{q}$}

In this section we study the nilpotent orbits in the orthogonal Lie algebras over $\mathbf{F}_{q}$ by extending the method in [2]. Let $V$ be a non-degenerate form space over $\overline{\mathbf{F}}_{q}$. An isomorphism class of form modules on $V$ over $\overline{\mathbf{F}}_{q}$ may decompose into several isomorphism classes over $\mathbf{F}_{q}$.

Proposition 4.1. Let $W$ be a form module $\left(\lambda_{1}\right)_{\chi\left(\lambda_{1}\right)}^{m_{1}}\left(\lambda_{2}\right)_{\chi\left(\lambda_{2}\right)}^{m_{2}} \cdots\left(\lambda_{s}\right)_{\chi\left(\lambda_{s}\right)}^{m_{s}}$ on the form space $V$.

(i) Assume $V$ is defective. The isomorphism class of $W$ over $\overline{\boldsymbol{F}}_{q}$ decomposes into at most $2^{n_{1}}$ isomorphism classes over $\boldsymbol{F}_{q}$, where $n_{1}$ is the cardinality of $\{1 \leq i \leq$ $\left.s-1 \mid \chi\left(\lambda_{i}\right)+\chi\left(\lambda_{i+1}\right) \leq \lambda_{i}, \chi\left(\lambda_{i}\right) \neq \lambda_{i} / 2\right\}$.

(ii) Assume $V$ is non-defective. The isomorphism class of $W$ over $\overline{\boldsymbol{F}}_{q}$ decomposes into at most $2^{n_{2}}$ isomorphism classes over $\boldsymbol{F}_{q}$, where $n_{2}$ is the cardinality of $\{1 \leq$ $\left.i \leq s \mid \chi\left(\lambda_{i}\right)+\chi\left(\lambda_{i+1}\right) \leq \lambda_{i}, \chi\left(\lambda_{i}\right) \neq \lambda_{i} / 2\right\}$ (here define $\chi\left(\lambda_{s+1}\right)=0$ ).

Note that we have two types of non-defective form spaces of dimension $2 n$ over $\mathbf{F}_{q}, V^{+}$with a quadratic form of Witt index $n$ and $V^{-}$with a quadratic form of Witt index $n-1$. We define $O_{2 n}^{+}\left(\mathbf{F}_{q}\right)$ (resp. $\left.O_{2 n}^{-}\left(\mathbf{F}_{q}\right)\right)$ to be $O\left(V^{+}\right)$ (resp. $O\left(V^{-}\right)$) and $\mathfrak{o}_{2 n}^{+}\left(\mathbf{F}_{q}\right)$ (resp. $\mathfrak{o}_{2 n}^{-}\left(\mathbf{F}_{q}\right)$ ) to be $\mathfrak{o}\left(V^{+}\right)$(resp. $\mathfrak{o}\left(V^{-}\right)$). Let $S O_{2 n}^{+}\left(\mathbf{F}_{q}\right)=O_{2 n}^{+}\left(\mathbf{F}_{q}\right) \cap S O_{2 n}\left(\overline{\mathbf{F}}_{q}\right)$. A form module on $V^{+}$(resp. $V^{-}$) has orthogonal decomposition $W_{l_{1}}^{\epsilon_{1}}\left(\lambda_{1}\right) \oplus \cdots \oplus W_{l_{k}}^{\epsilon_{k}}\left(\lambda_{k}\right)$ with $\#\left\{1 \leq i \leq k \mid \epsilon_{i}=\delta\right\}$ being even (resp. odd).

Corollary 4.2. (i) The nilpotent $O_{2 n+1}\left(\overline{\boldsymbol{F}}_{q}\right)$-orbit $\left(\lambda_{1}\right)_{\chi\left(\lambda_{1}\right)}^{m_{1}} \cdots\left(\lambda_{s}\right)_{\chi\left(\lambda_{s}\right)}^{m_{s}}$ in $\mathfrak{o}_{2 n+1}\left(\overline{\boldsymbol{F}}_{q}\right)$ decomposes into at most $2^{n_{1}} O_{2 n+1}\left(\boldsymbol{F}_{q}\right)$-orbits in $\mathfrak{o}_{2 n+1}\left(\boldsymbol{F}_{q}\right)$.

(ii) If $\chi\left(\lambda_{i}\right)=\lambda_{i} / 2, i=1, \ldots, s$, the nilpotent $O_{2 n}\left(\overline{\boldsymbol{F}}_{q}\right)$-orbit $\left(\lambda_{1}\right)_{\chi\left(\lambda_{1}\right)}^{m_{1}} \cdots\left(\lambda_{s}\right)_{\chi\left(\lambda_{s}\right)}^{m_{s}}$ in $\mathfrak{o}_{2 n}\left(\overline{\boldsymbol{F}}_{q}\right)$ remains as one $O_{2 n}^{+}\left(\boldsymbol{F}_{q}\right)$-orbit in $\mathfrak{o}_{2 n}^{+}\left(\boldsymbol{F}_{q}\right)$; otherwise, it decomposes into at most $2^{n_{2}-1} O_{2 n}^{+}\left(\boldsymbol{F}_{q}\right)$-orbits in $\mathfrak{o}_{2 n}^{+}\left(\boldsymbol{F}_{q}\right)$ and at most $2^{n_{2}-1} O_{2 n}^{-}\left(\boldsymbol{F}_{q}\right)$-orbits in $\mathfrak{o}_{2 n}^{-}\left(\boldsymbol{F}_{q}\right)$.

Here $n_{1}, n_{2}$ are as in Proposition 4.1

Remark 4.3. In Corollary 4.2(ii), if $\chi\left(\lambda_{i}\right)=\lambda_{i} / 2, i=1, \ldots, s$, then $n$ is even; if $\chi\left(\lambda_{i}\right) \neq \lambda_{i} / 2$ for some $i$, then $n_{2} \geq 1$.

Before we prove Proposition 4.1, we need the following lemma.

Lemma 4.4. (i) Assume $k \geq m$ and $l \geq m$. We have $W_{l}^{0}(k) \oplus D(m) \cong W_{l}^{\delta}(k) \oplus$ $D(m)$ if and only if $l+m>k$.

(ii) Assume $m>k$. We have $D(m) \oplus W_{k}^{0}(k) \cong D(m) \oplus W_{k}^{\delta}(k)$.

(iii) Assume $l_{1} \geq l_{2}, \lambda_{1}-l_{1} \geq \lambda_{2}-l_{2}$. If $l_{1}+l_{2}>\lambda_{1}$, then $W_{l_{1}}^{0}\left(\lambda_{1}\right) \oplus W_{l_{2}}^{0}\left(\lambda_{2}\right) \cong$ $W_{l_{1}}^{\delta}\left(\lambda_{1}\right) \oplus W_{l_{2}}^{\delta}\left(\lambda_{2}\right)$ and $W_{l_{1}}^{0}\left(\lambda_{1}\right) \oplus W_{l_{2}}^{\delta}\left(\lambda_{2}\right) \cong W_{l_{1}}^{\delta}\left(\lambda_{1}\right) \oplus W_{l_{2}}^{0}\left(\lambda_{2}\right)$. 
(iv) Assume $l_{1} \geq l_{2}, \lambda_{1}-l_{1} \geq \lambda_{2}-l_{2}$. If $l_{1}+l_{2} \leq \lambda_{1}$, then $W_{l_{1}}^{\epsilon_{1}}\left(\lambda_{1}\right) \oplus W_{l_{2}}^{\epsilon_{2}}\left(\lambda_{2}\right) \cong$ $W_{l_{1}}^{\epsilon_{1}^{\prime}}\left(\lambda_{1}\right) \oplus W_{l_{2}}^{\epsilon_{2}^{\prime}}\left(\lambda_{2}\right)$ if and only if $\epsilon_{1}=\epsilon_{1}^{\prime}, \epsilon_{2}=\epsilon_{2}^{\prime}$, where $\epsilon_{i}, \epsilon_{i}^{\prime}=0$ or $\delta, i=1,2$.

Proof. We only prove (i). (ii)-(iv) are proved similarly. Take $v_{1}, w_{1}$ and $v_{2}, w_{2}$ such that $W_{l}^{0}(k) \oplus D(m)=A v_{1} \oplus A w_{1} \oplus A v_{2} \oplus A w_{2}$ and $\psi\left(v_{1}\right)=t^{2-2 l}, \psi\left(w_{1}\right)=$ $0, \varphi\left(v_{1}, w_{1}\right)=t^{1-k}, \psi\left(v_{2}\right)=t^{2-2 m}, \psi\left(w_{2}\right)=0, \varphi\left(v_{2}, w_{2}\right)=t^{2-m}, \varphi\left(v_{1}, v_{2}\right)=$ $\varphi\left(v_{1}, w_{2}\right)=\varphi\left(w_{1}, v_{2}\right)=\varphi\left(w_{1}, w_{2}\right)=0$. Similarly, take $v_{1}^{\prime}, w_{1}^{\prime}$ and $v_{2}^{\prime}, w_{2}^{\prime}$ such that $W_{l}^{\delta}(k) \oplus D(m)=A v_{1}^{\prime} \oplus A w_{1}^{\prime} \oplus A v_{2}^{\prime} \oplus A w_{2}^{\prime}$ and $\psi\left(v_{1}^{\prime}\right)=t^{2-2 l}, \psi\left(w_{1}^{\prime}\right)=$ $\delta t^{2 l-2 k}, \varphi\left(v_{1}^{\prime}, w_{1}^{\prime}\right)=t^{1-k}, \psi\left(v_{2}^{\prime}\right)=t^{2-2 m}, \psi\left(w_{2}^{\prime}\right)=0, \varphi\left(v_{2}^{\prime}, w_{2}^{\prime}\right)=t^{2-m}, \varphi\left(v_{1}^{\prime}, v_{2}^{\prime}\right)=$ $\varphi\left(v_{1}^{\prime}, w_{2}^{\prime}\right)=\varphi\left(w_{1}^{\prime}, v_{2}^{\prime}\right)=\varphi\left(w_{1}^{\prime}, w_{2}^{\prime}\right)=0$.

We have $W_{l}^{0}(k) \oplus D(m) \cong W_{l}^{\delta}(k) \oplus D(m)$ if and only if there exists an $A$-module isomorphism $g: V \rightarrow V$ such that $\psi(g v)=\psi(v)$ and $\varphi(g v, g w)=\varphi(v, w)$ for any $v, w \in V$. Assume

$$
\begin{aligned}
g v_{j} & =\sum_{i=0}^{k-1}\left(a_{j, i} t^{i} v_{1}^{\prime}+b_{j, i} t^{i} w_{1}^{\prime}\right)+\sum_{i=0}^{m-1} c_{j, i} t^{i} v_{2}^{\prime}+\sum_{i=0}^{m-2} d_{j, i} t^{i} w_{2}^{\prime}, \\
g w_{j} & =\sum_{i=0}^{k-1}\left(e_{j, i} t^{i} v_{1}^{\prime}+f_{j, i} t^{i} w_{1}^{\prime}\right)+\sum_{i=0}^{m-1} g_{j, i} t^{i} v_{2}^{\prime}+\sum_{i=0}^{m-2} h_{j, i} t^{i} w_{2}^{\prime}, j=1,2 .
\end{aligned}
$$

Then $W_{l}^{0}(k) \oplus D(m) \cong W_{l}^{\delta}(k) \oplus D(m)$ if and only if the equations $\psi\left(g v_{i}\right)=\psi\left(v_{i}\right)$, $\psi\left(g w_{i}\right)=\psi\left(w_{i}\right), \varphi\left(g v_{i}, g v_{j}\right)=\varphi\left(v_{i}, v_{j}\right), \varphi\left(g v_{i}, g w_{j}\right)=\varphi\left(v_{i}, w_{j}\right), \varphi\left(g w_{i}, g w_{j}\right)=$ $\varphi\left(w_{i}, w_{j}\right)$ have solutions.

If $l+m \leq k$, some equations are $e_{1,2 l-k-1}^{2}+e_{1,2 l-k-1}=\delta\left(l \neq \frac{k+1}{2}\right)$ or $a_{1,0}^{2}+$ $a_{1,0} b_{1,0}=1, e_{1,0}^{2}+e_{1,0} f_{1,0}=\delta, a_{1,0} f_{1,0}+b_{1,0} e_{1,0}=1\left(l=\frac{k+1}{2}\right)$. By the definition of $\delta$, this has no solution for $e_{1,2 l-k-1}$ or $a_{1,0}, b_{1,0}, e_{1,0}, f_{1,0}$, which implies that $W_{l}^{0}(k) \oplus D(m) \varsubsetneqq W_{l}^{\delta}(k) \oplus D(m)$.

If $l+m>k$, let $g v_{1}=v_{1}^{\prime}, g w_{1}=w_{1}^{\prime}+\sqrt{\delta} t^{l+m-k-1} v_{2}^{\prime}, g v_{2}=v_{2}^{\prime}, g w_{2}=w_{2}^{\prime}+\sqrt{\delta} t^{l} v_{1}^{\prime}$. This is a solution for the equations. It follows that $W_{l}^{0}(k) \oplus D(m) \cong W_{l}^{\delta}(k) \oplus$ $D(m)$.

Proof of Proposition 4.1. We prove (i). One can prove (ii) similarly. By the classification in subsection 2.4, we can rewrite the symbol as

$\left(\lambda_{1}\right)_{\chi\left(\lambda_{1}\right)}^{2}\left(\lambda_{2}\right)_{\chi\left(\lambda_{2}\right)}^{2} \cdots\left(\lambda_{k}\right)_{\chi\left(\lambda_{k}\right)}^{2}\left(\lambda_{k+1}\right)_{\lambda_{k+1}}\left(\lambda_{k+1}-1\right)_{\left(\lambda_{k+1}-1\right)}\left(\lambda_{k+2}\right)_{\lambda_{k+2}}^{2} \cdots\left(\lambda_{k+l}\right)_{\lambda_{k+l}}^{2}$, where $\lambda_{1} \geq \cdots \geq \lambda_{k+1}>\lambda_{k+2} \geq \cdots \geq \lambda_{k+l}, \chi\left(\lambda_{i}\right) \geq \chi\left(\lambda_{i+1}\right)$ and $\lambda_{i}-\chi\left(\lambda_{i}\right) \geq$ $\lambda_{i+1}-\chi\left(\lambda_{i+1}\right)$ for $1 \leq i \leq k$ (by abuse of notation, we still use $\lambda$ ), $l \geq 1$. A representative $W$ for this isomorphism class over $\overline{\mathbf{F}}_{q}$ is $W_{\chi\left(\lambda_{1}\right)}\left(\lambda_{1}\right) \oplus \cdots \oplus W_{\chi\left(\lambda_{k}\right)}\left(\lambda_{k}\right) \oplus$ $D\left(\lambda_{k+1}\right) \oplus W_{\lambda_{k+2}}\left(\lambda_{k+2}\right) \oplus \cdots \oplus W_{\lambda_{k+l}}\left(\lambda_{k+l}\right)$. By Proposition 3.1 and Remark 3.2. in order to study the isomorphism classes into which the isomorphism class of $W$ over $\overline{\mathbf{F}}_{q}$ decomposes over $\mathbf{F}_{q}$, it is enough to study the isomorphism classes of form modules of the form $W_{\chi\left(\lambda_{1}\right)}^{\epsilon_{1}}\left(\lambda_{1}\right) \oplus \cdots \oplus W_{\chi\left(\lambda_{k}\right)}^{\epsilon_{k}}\left(\lambda_{k}\right) \oplus D\left(\lambda_{k+1}\right) \oplus W_{\lambda_{k+2}}^{\epsilon_{k+2}}\left(\lambda_{k+2}\right) \oplus$ $\cdots \oplus W_{\lambda_{k+l}}^{\epsilon_{k+l}}\left(\lambda_{k+l}\right)$, where $\epsilon_{i}=0$ or $\delta$. Thus it suffices to show that modules of the above form have at most $2^{n_{1}}$ isomorphism classes.

We have $n_{1}=\#\left\{1 \leq i \leq k \mid \chi\left(\lambda_{i}\right)+\chi\left(\lambda_{i+1}\right) \leq \lambda_{i}, \chi\left(\lambda_{i}\right) \neq \lambda_{i} / 2\right\}$. Suppose $i_{1}, i_{2}, \ldots, i_{n_{1}}$ are such that $1 \leq i_{j} \leq k, \chi\left(\lambda_{i_{j}}\right)+\chi\left(\lambda_{i_{j}+1}\right) \leq \lambda_{i_{j}}, \chi\left(\lambda_{i_{j}}\right) \neq \lambda_{i_{j}} / 2, j=$ $1, \ldots, n_{1}$. Then using Lemma 4.4 one can easily show that a module of the above form is isomorphic to one of the following modules: $V_{1}^{\epsilon_{1}} \oplus \cdots \oplus V_{n_{1}}^{\epsilon_{n_{1}}} \oplus V_{n_{1}+1}$, where $V_{t}^{\epsilon_{t}}=W_{\chi\left(\lambda_{i_{t-1}+1}\right)}^{0}\left(\lambda_{i_{t-1}+1}\right) \oplus \cdots \oplus W_{\chi\left(\lambda_{i_{t}-1}\right)}^{0}\left(\lambda_{i_{t}-1}\right) \oplus W_{\chi\left(\lambda_{i_{t}}\right)}^{\epsilon_{t}}\left(\lambda_{i_{t}}\right), t=1, \ldots, n_{1}$, 
$i_{0}=0, \epsilon_{t}=0$ or $\delta$ and $V_{n_{1}+1}=W_{\chi\left(\lambda_{n_{1}+1}\right)}^{0}\left(\lambda_{i_{n_{1}}+1}\right) \oplus \cdots \oplus W_{\chi\left(\lambda_{k}\right)}^{0}\left(\lambda_{k}\right) \oplus D\left(\lambda_{k+1}\right) \oplus$ $W_{\lambda_{k+2}}^{0}\left(\lambda_{k+2}\right) \oplus \cdots \oplus W_{\lambda_{k+l}}^{0}\left(\lambda_{k+l}\right)$. Thus (i) is proved.

\section{Number of Nilpotent orbits OVER $\mathbf{F}_{q}$}

5.1. In this subsection we recall Spaltenstein's parametrization of nilpotent orbits by pairs of partitions in $\mathfrak{o}\left(\overline{\mathbf{F}}_{q}\right)$ (see $\left.[9]\right)$.

For $\mathfrak{o}_{2 n+1}\left(\overline{\mathbf{F}}_{q}\right)$, the orbit

$$
\left(\lambda_{1}\right)_{\chi\left(\lambda_{1}\right)}^{2} \cdots\left(\lambda_{k}\right)_{\chi\left(\lambda_{k}\right)}^{2}\left(\lambda_{k+1}\right)_{\lambda_{k+1}}\left(\lambda_{k+1}-1\right)_{\left(\lambda_{k+1}-1\right)}\left(\lambda_{k+2}\right)_{\lambda_{k+2}}^{2} \cdots\left(\lambda_{k+l}\right)_{\lambda_{k+l}}^{2}
$$

is written as

$$
\begin{array}{r}
\left(\alpha_{1}+\beta_{1}\right)_{\left(\alpha_{1}+1\right)}^{2} \cdots\left(\alpha_{k}+\beta_{k}\right)_{\left(\alpha_{k}+1\right)}^{2}\left(\alpha_{k+1}+1\right)_{\left(\alpha_{k+1}+1\right)}\left(\alpha_{k+1}\right)_{\alpha_{k+1}}\left(\alpha_{k+2}\right)_{\alpha_{k+2}}^{2} \\
\cdots\left(\alpha_{k+l}\right)_{\alpha_{k+l}}^{2}
\end{array}
$$

and the corresponding pair of partitions is $(\alpha, \beta)$, where $\alpha=\left(\alpha_{1} \ldots, \alpha_{k+l}\right)$ and $\beta=\left(\beta_{1}, \ldots, \beta_{k}\right)$ satisfy $\alpha_{1} \geq \cdots \geq \alpha_{k+l} \geq 0, \beta_{1} \geq \cdots \geq \beta_{k} \geq 1$ and $|\alpha|+|\beta|=n$.

For $\mathfrak{o}_{2 n}\left(\overline{\mathbf{F}}_{q}\right)$, the orbit

is written as

$$
\left(\lambda_{1}\right)_{\chi\left(\lambda_{1}\right)}^{2} \cdots\left(\lambda_{k}\right)_{\chi\left(\lambda_{k}\right)}^{2}
$$

$$
\left(\alpha_{1}+\beta_{1}\right)_{\alpha_{1}}^{2} \cdots\left(\alpha_{k}+\beta_{k}\right)_{\alpha_{k}}^{2}
$$

and the corresponding pair of partitions is $(\alpha, \beta)$, where $\alpha=\left(\alpha_{1}, \ldots, \alpha_{k}\right)$ and $\beta=\left(\beta_{1}, \ldots, \beta_{k}\right)$ satisfy $\alpha_{1} \geq \cdots \geq \alpha_{k} \geq 1, \beta_{1} \geq \cdots \geq \beta_{k} \geq 0$ and $|\alpha|+|\beta|=n$.

5.2. In this subsection we study the number of nilpotent orbits over $\mathbf{F}_{q}$. Denote by $p_{2}(n)$ the cardinality of the set of pairs of partitions $(\alpha, \beta)$ such that $|\alpha|+|\beta|=n$ and $p(k)$ the number of partitions of the integer $k$.

Proposition 5.1. (i) The number of nilpotent $O_{2 n+1}\left(\boldsymbol{F}_{q}\right)$-orbits in $\mathfrak{o}_{2 n+1}\left(\boldsymbol{F}_{q}\right)$ is at most $p_{2}(n)$.

(ii) The number of nilpotent $O_{2 n}^{+}\left(\boldsymbol{F}_{q}\right)$-orbits in $\mathfrak{o}_{2 n}^{+}\left(\boldsymbol{F}_{q}\right)$ is at most $\frac{1}{2} p_{2}(n)$ if $n$ is odd and is at most $\frac{1}{2}\left(p_{2}(n)+p\left(\frac{n}{2}\right)\right)$ if $n$ is even.

Proof. (i) The set of nilpotent orbits in $\mathfrak{o}_{2 n+1}\left(\overline{\mathbf{F}}_{q}\right)$ is mapped bijectively to the set $\left\{(\alpha, \beta)|| \alpha|+| \beta \mid=n, \beta_{i} \leq \alpha_{i}+2\right\}:=\Delta([9])$. By Corollary 4.2(i), a nilpotent orbit in $\mathfrak{o}_{2 n+1}\left(\overline{\mathbf{F}}_{q}\right)$ corresponding to $(\alpha, \beta) \in \Delta, \alpha=\left(\alpha_{1}, \ldots, \alpha_{s}\right), \beta=\left(\beta_{1}, \ldots, \beta_{t}\right)$ splits into at most $2^{n_{1}}$ orbits in $\mathfrak{o}_{2 n+1}\left(\mathbf{F}_{q}\right)$, where $n_{1}=\#\left\{1 \leq i \leq t \mid \alpha_{i+1}+2 \leq \beta_{i}<\alpha_{i}+2\right\}$. We associate to this orbit $2^{n_{1}}$ pairs of partitions as follows. Suppose $r_{1}, r_{2}, \ldots, r_{n_{1}}$ are such that $\alpha_{r_{i}+1}+2 \leq \beta_{r_{i}}<\alpha_{r_{i}}+2, i=1, \ldots, n_{1}$. Let

$$
\begin{aligned}
& \alpha^{1, i}=\left(\alpha_{r_{i-1}+1}, \ldots, \alpha_{r_{i}}\right), \beta^{1, i}=\left(\beta_{r_{i-1}+1}, \ldots, \beta_{r_{i}}\right), \\
& \alpha^{2, i}=\left(\beta_{r_{i-1}+1}-2, \ldots, \beta_{r_{i}}-2\right), \beta^{2, i}=\left(\alpha_{r_{i-1}+1}+2, \ldots, \alpha_{r_{i}}+2\right), i=1, \ldots, n_{1}, \\
& \alpha^{n_{1}+1}=\left(\alpha_{r_{n_{1}}+1}, \ldots, \alpha_{s}\right), \beta^{n_{1}+1}=\left(\beta_{r_{n_{1}}+1}, \ldots, \beta_{t}\right) .
\end{aligned}
$$

We associate to $(\alpha, \beta)$ the pairs of partitions $\left(\tilde{\alpha}^{\epsilon_{1}, \ldots, \epsilon_{n_{1}}}, \tilde{\beta}^{\epsilon_{1}, \ldots, \epsilon_{n_{1}}}\right)$,

$$
\tilde{\alpha}^{\epsilon_{1}, \ldots, \epsilon_{n_{1}}}=\left(\alpha^{\epsilon_{1}, 1}, \ldots, \alpha^{\epsilon_{n_{1}}, n_{1}}, \alpha^{n_{1}+1}\right), \tilde{\beta}^{\epsilon_{1}, \ldots, \epsilon_{n_{1}}}=\left(\beta^{\epsilon_{1}, 1}, \ldots, \beta^{\epsilon_{n_{1}}, n_{1}}, \beta^{n_{1}+1}\right),
$$

where $\epsilon_{i} \in\{1,2\}, i=1, \ldots, n_{1}$.

Notice that the pairs of partitions $\left(\tilde{\alpha}^{\epsilon_{1}, \ldots, \epsilon_{n_{1}}}, \tilde{\beta}^{\epsilon_{1}, \ldots, \epsilon_{n_{1}}}\right)$ are distinct and among them only $(\alpha, \beta)=\left(\tilde{\alpha}^{1, \ldots, 1}, \tilde{\beta}^{1, \ldots, 1}\right)$ is in $\Delta$. One can verify that the set of all pairs

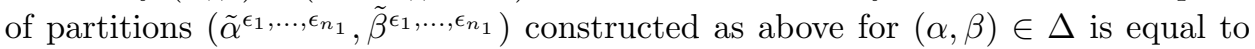


the set $\{(\alpha, \beta)|| \alpha|+| \beta \mid=n\}$, which has cardinality $p_{2}(n)$. But the number of nilpotent orbits in $\mathfrak{o}_{2 n+1}\left(\mathbf{F}_{q}\right)$ is no greater than the cardinality of the former set. Thus (i) is proved.

(ii) Similarly, the set of nilpotent orbits in $\mathfrak{o}_{2 n}\left(\overline{\mathbf{F}}_{q}\right)$ is mapped bijectively to the set $\left\{(\alpha, \beta)|| \alpha|+| \beta \mid=n, \beta_{i} \leq \alpha_{i}\right\}:=\Delta^{\prime}([9])$. By Corollary 4.2 (ii), a nilpotent orbit in $\mathfrak{o}_{2 n}\left(\overline{\mathbf{F}}_{q}\right)$ corresponding to $(\alpha, \beta) \in \Delta^{\prime}$ with $\alpha=\left(\alpha_{1}, \alpha_{2}, \ldots, \alpha_{s}\right), \beta=\left(\beta_{1}, \beta_{2}, \ldots, \beta_{s}\right)$ and $\alpha \neq \beta$ splits into at most $2^{n_{2}-1}$ orbits in $\mathfrak{o}_{2 n}^{+}\left(\mathbf{F}_{q}\right)$, where $n_{2}=\#\left\{i \mid \alpha_{i+1} \leq \beta_{i}<\right.$ $\left.\alpha_{i}\right\}-1$. We associate to this orbit $2^{n_{2}-1}$ pairs of partitions as follows. We can assume $\alpha_{s} \neq 0$. Suppose $r_{1}, r_{2}, \ldots, r_{n_{2}}$ are such that $\alpha_{r_{i}+1} \leq \beta_{r_{i}}<\alpha_{r_{i}}, i=$ $1, \ldots, n_{2}$. Let

$$
\begin{aligned}
& \alpha^{1, i}=\left(\alpha_{r_{i-1}+1}, \ldots, \alpha_{r_{i}}\right), \beta^{1, i}=\left(\beta_{r_{i-1}+1}, \ldots, \beta_{r_{i}}\right), \\
& \alpha^{2, i}=\left(\beta_{r_{i-1}+1}, \ldots, \beta_{r_{i}}\right), \beta^{2, i}=\left(\alpha_{r_{i-1}+1}, \ldots, \alpha_{r_{i}}\right), i=1, \ldots, n_{2}, \\
& \alpha^{n_{2}+1}=\left(\alpha_{r_{n_{2}}+1}, \ldots, \alpha_{s}\right), \beta^{n_{2}+1}=\left(\beta_{r_{n_{2}}+1}, \ldots, \beta_{s}\right) .
\end{aligned}
$$

We have $2^{n_{2}}$ distinct pairs of partitions $\left(\tilde{\alpha}^{\epsilon_{1}, \ldots, \epsilon_{n_{2}}}, \tilde{\beta}^{\epsilon_{1}, \ldots, \epsilon_{n_{2}}}\right)$,

$$
\tilde{\alpha}^{\epsilon_{1}, \ldots, \epsilon_{n_{2}}}=\left(\alpha^{\epsilon_{1}, 1}, \ldots, \alpha^{\epsilon_{n_{2}}, n_{2}}, \alpha^{n_{2}+1}\right), \tilde{\beta}^{\epsilon_{1}, \ldots, \epsilon_{n_{2}}}=\left(\beta^{\epsilon_{1}, 1}, \ldots, \beta^{\epsilon_{n_{2}}, n_{2}}, \beta^{n_{2}+1}\right),
$$

where $\epsilon_{i} \in\{1,2\}, i=1, \ldots, n_{2}$. We show that in these pairs of partitions $\left(\alpha^{\prime}, \beta^{\prime}\right)$ appears if and only if $\left(\beta^{\prime}, \alpha^{\prime}\right)$ appears. In fact we have $\alpha_{i}=\beta_{i}$, for $i>r_{n_{2}}$, which implies $\alpha^{n_{2}+1}=\beta^{n_{2}+1}$. Thus we have

$$
\left(\tilde{\alpha}^{\epsilon_{1}+1(\bmod 2), \ldots, \epsilon_{n_{2}}+1(\bmod 2)}, \tilde{\beta}^{\epsilon_{1}+1(\bmod 2), \ldots, \epsilon_{n_{2}}+1(\bmod 2)}\right)=\left(\tilde{\beta}^{\epsilon_{1}, \ldots, \epsilon_{n_{2}}}, \tilde{\alpha}^{\epsilon_{1}, \ldots, \epsilon_{n_{2}}}\right) .
$$

Hence we can identify $\left(\alpha^{\prime}, \beta^{\prime}\right)$ with $\left(\beta^{\prime}, \alpha^{\prime}\right)$, and then associate $2^{n_{2}-1}$ pairs of partitions to the nilpotent orbit corresponding to $(\alpha, \beta)$.

One can verify that the set of all pairs of partitions associated to $(\alpha, \beta) \in \Delta^{\prime}$ as above is in bijection with the set of pairs of partitions $(\alpha, \beta)$ such that $|\alpha|+|\beta|=n$ with $(\alpha, \beta)$ identified with $(\beta, \alpha)$, which has cardinality $\frac{1}{2} p_{2}(n)$ if $n$ is odd and $\frac{1}{2}\left(p_{2}(n)+p\left(\frac{n}{2}\right)\right)$ if $n$ is even. Thus (ii) follows.

Corollary 5.2. The number of nilpotent $S O_{2 n}^{+}\left(\boldsymbol{F}_{q}\right)$-orbits in $\mathfrak{o}_{2 n}^{+}\left(\boldsymbol{F}_{q}\right)$ is at most $\frac{1}{2} p_{2}(n)$ if $n$ is odd and is at most $\frac{1}{2}\left(p_{2}(n)+3 p\left(\frac{n}{2}\right)\right)$ if $n$ is even.

Proof. We show that the $O_{2 n}^{+}\left(\mathbf{F}_{q}\right)$ orbits that split into two $S_{2 n}^{+}\left(\mathbf{F}_{q}\right)$-orbits are exactly the orbits corresponding to the pairs of partitions of the form $(\alpha, \alpha)$. The number of these orbits is $p\left(\frac{n}{2}\right)$. Let $x$ be a nilpotent element in $\mathfrak{o}_{2 n}^{+}\left(\mathbf{F}_{q}\right)$. The $O_{2 n}^{+}\left(\mathbf{F}_{q}\right)$-orbit of $x$ splits into two $S O_{2 n}^{+}\left(\mathbf{F}_{q}\right)$-orbits if and only if the centralizer $Z_{O_{2 n}^{+}\left(\mathbf{F}_{q}\right)}(x) \subset S O_{2 n}^{+}\left(\mathbf{F}_{q}\right)$. It is enough to show that for an indecomposable module $V, Z_{O_{2 n}^{+}\left(\mathbf{F}_{q}\right)}(V) \subset S O_{2 n}^{+}\left(\mathbf{F}_{q}\right)$ if and only if $\chi(m) \leq \frac{1}{2} m$, for all $m \in \mathbb{N}$.

Assume $V=W_{l}^{0}(m)$ or $W_{l}^{\delta}(m), l \geq \frac{m+1}{2}$. Let $\epsilon=0$ or $\delta$ and $v_{1}^{\epsilon}, v_{2}^{\epsilon}$ be such that $W_{l}^{\epsilon}(m)=A v_{1}^{\epsilon} \oplus A v_{2}^{\epsilon}$ and $\psi\left(v_{1}^{\epsilon}\right)=t^{2-2 l}, \psi\left(v_{2}^{\epsilon}\right)=\epsilon t^{-2 m+2 l}, \varphi\left(v_{1}^{\epsilon}, v_{2}^{\epsilon}\right)=t^{1-m}$. Let $w_{2}^{\epsilon}=v_{2}^{\epsilon}+t^{2 l-1-m} v_{1}^{\epsilon}$. Define $u^{\epsilon}$ by $u^{\epsilon}\left(a_{1} v_{1}^{\epsilon}+a_{2} v_{2}^{\epsilon}\right)=a_{1} v_{1}^{\epsilon}+a_{2} w_{2}^{\epsilon}$. Then $u^{\epsilon} \epsilon$ $Z_{O_{2 n}^{+}\left(\mathbf{F}_{q}\right)}\left(W_{l}^{\epsilon}(m)\right)$, but $u^{\epsilon} \notin S O_{2 n}^{+}\left(\mathbf{F}_{q}\right)$ (see [2]). This shows that $Z_{O_{2 n}^{+}\left(\mathbf{F}_{q}\right)}(V) \nsubseteq$ $S O_{2 n}^{+}\left(\mathbf{F}_{q}\right)$.

Assume $V=W_{l}^{0}(m), l=m / 2$. Let $v_{1}, v_{2}$ be such that $W_{l}^{0}(m)=A v_{1} \oplus A v_{2}$ and $\psi\left(v_{1}\right)=t^{2-m}, \psi\left(v_{2}\right)=0, \varphi\left(v_{1}, v_{2}\right)=t^{1-m}$. Let $W$ be the subspace of $V$ spanned by $t^{\frac{m}{2}} v_{1}, t^{\frac{m}{2}+1} v_{1}, \ldots, t^{m-1} v_{1}, t^{\frac{m}{2}} v_{2}, t^{\frac{m}{2}+1} v_{2}, \ldots, t^{m-1} v_{2}$. Then $W$ is a maximal totally singular subspace in $V$ and $Z_{O_{2 n}^{+}\left(\mathbf{F}_{q}\right)}(V)$ stabilizes $W$. Hence $Z_{O_{2 n}^{+}\left(\mathbf{F}_{q}\right)}(V) \subset S O_{2 n}^{+}\left(\mathbf{F}_{q}\right)$. 


\section{SPRINGER CORRESPONDENCE}

Throughout subsections 6.1 6.5, let $G$ be a connected adjoint algebraic group of type $B_{r}, C_{r}$ or $D_{r}$ over $\mathbf{k}$ and let $\mathfrak{g}$ be the Lie algebra of $G$. Fix a Borel subgroup $B$ of $G$ with Levi decomposition $B=T U$. Let $\mathfrak{b}, \mathfrak{t}$ and $\mathfrak{n}$ be the Lie algebra of $B, T$ and $U$, respectively. Let $\mathcal{B}$ be the variety of Borel subgroups of $G$.

We construct in this section the Springer correspondence for the Lie algebra $\mathfrak{g}$ following 4, 5. The construction and proofs are essentially the same as (actually simpler than) those for the unipotent case in 4, 5]. For completeness, we include the proofs here. We only point out that Lemma 6.2 is essential for the construction. The lemma is probably well-known for which we include an elementary proof.

6.1. Let $Z$ be the variety $\left\{\left(x, B_{1}, B_{2}\right) \in \mathfrak{g} \times \mathcal{B} \times \mathcal{B} \mid x \in \mathfrak{b}_{1} \cap \mathfrak{b}_{2}\right\}$ and $Z^{\prime}$ the Steinberg variety [15] $\left\{\left(x, B_{1}, B_{2}\right) \in \mathfrak{g} \times \mathcal{B} \times \mathcal{B} \mid x \in \mathfrak{n}_{1} \cap \mathfrak{n}_{2}\right\}$. Denote $r$ as the dimension of $T$. Let $\mathrm{c}$ be a nilpotent orbit in $\mathfrak{g}$. A stronger version of the following lemma in the group case is due to Springer, Steinberg and Spaltenstein (see for example [10]). We include a proof for the Lie algebra case here.

Lemma 6.1. (i) We have $\operatorname{dim}(\mathrm{c} \cap \mathfrak{n}) \leq \frac{1}{2} \operatorname{dim} \mathrm{c}$.

(ii) Given $x \in \mathrm{c}$, we have $\operatorname{dim}\left\{B_{1} \in \mathcal{B} \mid x \in \mathfrak{n}_{1}\right\} \leq(\operatorname{dim} G-r-\operatorname{dim} \mathrm{c}) / 2$.

(iii) We have $\operatorname{dim} Z=\operatorname{dim} G$ and $\operatorname{dim} Z^{\prime}=\operatorname{dim} G-r$.

Proof. We have a partition $Z=\cup_{\mathcal{O}} Z_{\mathcal{O}}$ according to the $G$-orbits $\mathcal{O}$ on $\mathcal{B} \times \mathcal{B}$ where $Z_{\mathcal{O}}=\left\{\left(x, B_{1}, B_{2}\right) \in Z \mid\left(B_{1}, B_{2}\right) \in \mathcal{O}\right\}$. Define in the same way a partition $Z^{\prime}=\bigcup_{\mathcal{O}} Z_{\mathcal{O}}^{\prime}$. Consider the maps from $Z_{\mathcal{O}}$ and $Z_{\mathcal{O}}^{\prime}$ to $\mathcal{O}:\left(x, B_{1}, B_{2}\right) \mapsto\left(B_{1}, B_{2}\right)$. We have $\operatorname{dim} Z_{\mathcal{O}}=\operatorname{dim}\left(\mathfrak{b}_{1} \cap \mathfrak{b}_{2}\right)+\operatorname{dim} \mathcal{O}=\operatorname{dim} G$ and $\operatorname{dim} Z_{\mathcal{O}}^{\prime}=\operatorname{dim}\left(\mathfrak{n}_{1} \cap \mathfrak{n}_{2}\right)+$ $\operatorname{dim} \mathcal{O}=\operatorname{dim} G-r$. Thus (iii) follows.

Let $Z^{\prime}(\mathrm{c})=\left\{\left(x, B_{1}, B_{2}\right) \in Z^{\prime} \mid x \in \mathrm{c}\right\} \subset Z^{\prime}$. From (iii), we have $\operatorname{dim} Z^{\prime}(\mathrm{c}) \leq$ $\operatorname{dim} G-r$. Consider the map $Z^{\prime}(\mathrm{c}) \rightarrow \mathrm{c},\left(x, B_{1}, B_{2}\right) \mapsto x$. We have $\operatorname{dim} Z^{\prime}(\mathrm{c})=$ $\operatorname{dim} \mathrm{c}+2 \operatorname{dim}\left\{B_{1} \in \mathcal{B} \mid x \in \mathfrak{n}_{1}\right\} \leq \operatorname{dim} G-r$. Thus (ii) follows.

Consider the variety $\left\{\left(x, B_{1}\right) \in \mathrm{c} \times \mathcal{B} \mid x \in \mathfrak{n}_{1}\right\}$. By projecting it to the first coordinate, and using (ii), we see that it has dimension $\leq(\operatorname{dim} G-r+\operatorname{dim} \mathrm{c}) / 2$. If we project it to the second coordinate, we get $\operatorname{dim}(\mathrm{c} \cap \mathfrak{n})+\operatorname{dim} \mathcal{B} \leq(\operatorname{dim} G-r+\operatorname{dim} \mathrm{c}) / 2$ and (i) follows.

6.2. Recall that an element $x$ in $\mathfrak{g}$ is called regular if the connected centralizer $Z_{G}^{0}(x)$ in $G$ is a maximal torus of $G$.

Lemma 6.2. There exist regular semisimple elements in $\mathfrak{g}$ and they form an open dense subset in $\mathfrak{g}$.

Proof. We first show that regular semisimple elements exists in $\mathfrak{g}$.

(i) $G=S O(2 n+1)$. Let $V$ be a $2 n+1$-dimensional vector space equipped with a non-degenerate quadratic form $Q: V \rightarrow k$. Let $\langle$,$\rangle be the bilinear form$ associated to $Q$ defined by $\langle v, w\rangle=Q(v+w)+Q(v)+Q(w)$. Then $G$ is defined as $\{g \in G L(V) \mid Q(g v)=Q(v), \forall v \in V\}$. Since $Q$ is non-degenerate, we have $\operatorname{dim} V^{\perp}=1$. Let $V^{\perp}=\operatorname{span}\left\{v_{0}\right\}$, we have $g v_{0}=v_{0}$, for any $g \in G$. Hence the Lie algebra is $\left\{x \in \operatorname{End}(V) \mid\langle x v, v\rangle=0, \forall v \in V ; x v_{0}=0\right\}$. With respect to a suitable basis, we can assume $Q(v)=v^{t} B v$, where

$$
B=\left(\begin{array}{ccc}
0 & I_{n \times n} & 0 \\
0_{n \times n} & 0 & 0 \\
0 & 0 & 1
\end{array}\right)
$$


A maximal torus of $G$ is $T=\left\{\operatorname{diag}\left(t_{1}, t_{2}, \ldots, t_{n}, 1 / t_{1}, 1 / t_{2}, \ldots 1 / t_{n}, 1\right) \mid t_{i} \in k^{*}, i=\right.$ $1, \ldots, n\}$. The Lie algebra of $T$ is $\mathfrak{t}=\left\{\operatorname{diag}\left(x_{1}, x_{2}, \ldots, x_{n}, x_{1}, x_{2}, \ldots x_{n}, 0\right) \mid x_{i} \in\right.$ $k, i=1, \ldots, n\}$. Since every semisimple element in $\mathfrak{g}$ is conjugate to an element in $\mathfrak{t}$ under the adjoint action of $G$, it is enough to consider elements in $\mathfrak{t}$.

Let $x=\operatorname{diag}\left(x_{1}, x_{2}, \ldots, x_{n}, x_{1}, x_{2}, \ldots x_{n}, 0\right)$, where $x_{i} \neq x_{j}$, for any $i \neq j$ and $x_{i} \neq 0$ for any $i$ (such $x$ exists). It can be easily verified that $Z_{G}(x)$ consists of elements of the form

$$
g=\left(\begin{array}{ccc}
A_{1} & A_{2} & 0 \\
A_{3} & A_{4} & 0 \\
0 & 0 & 1
\end{array}\right)
$$

where $A_{i}=\operatorname{diag}\left(a_{i}^{1}, a_{i}^{2}, \ldots, a_{i}^{n}\right), i=1,2,3,4$, satisfy $a_{1}^{j} a_{3}^{j}=a_{2}^{j} a_{4}^{j}=0$ and $a_{1}^{j} a_{4}^{j}+$ $a_{2}^{j} a_{3}^{j}=1, j=1, \ldots, n$. Hence we see that $Z_{G}^{0}(x)=T$ and $x$ is regular.

(ii) $G$ is the adjoint group of type $C_{n}$. We have the following construction of $G$. Let $V$ be a $2 n$-dimensional vector space equipped with a non-degenerate symplectic form $\langle\rangle:, V \times V \rightarrow k$. Then $G$ is defined as

$$
\left\{(g, \lambda) \in G L(V) \times k^{*} \mid \forall v, w \in V,\langle g v, g w\rangle=\lambda\langle v, w\rangle\right\} /\left\{\left(\mu I, \mu^{2}\right) \mid \mu \in k^{*}\right\} .
$$

Hence the Lie algebra $\mathfrak{g}$ is

$$
\{(x, \lambda) \in \operatorname{End}(V) \times k \mid \forall v, w \in V,\langle x v, w\rangle+\langle v, x w\rangle=\lambda\langle v, w\rangle\} /\{(\mu I, 0) \mid \mu \in k\} .
$$

With respect to a suitable basis, we can assume $\langle v, w\rangle=v^{t} A w$ where

$$
A=\left(\begin{array}{cc}
0 & I_{n \times n} \\
I_{n \times n} & 0
\end{array}\right) .
$$

A maximal torus of $G$ is $T=\left\{\operatorname{diag}\left(t_{1}, t_{2}, \ldots, t_{n}, \lambda / t_{1}, \lambda / t_{2}, \ldots \lambda / t_{n}\right) \mid t_{i} \in k^{*}, i=\right.$ $\left.1, \ldots, n ; \lambda \in k^{*}\right\} /\left\{\left(\mu I, \mu^{2}\right) \mid \mu \in k^{*}\right\}$. The Lie algebra of $T$ is $\mathfrak{t}=\left\{\operatorname{diag}\left(x_{1}, x_{2}, \ldots, x_{n}\right.\right.$, $\left.\left.\lambda+x_{1}, \lambda+x_{2}, \ldots \lambda+x_{n}\right) \mid x_{i} \in k, i=1, \ldots, n ; \lambda \in k\right\} /\{(\mu I, 0) \mid \mu \in k\}$.

Let $x=\overline{\operatorname{diag}\left(x_{1}, x_{2}, \ldots, x_{n}, x_{1}+\lambda, x_{2}+\lambda, \ldots x_{n}+\lambda\right)} \in \mathfrak{t}$, where $x_{i} \neq x_{j}$, for any $i \neq j$ and $\lambda \neq x_{i}+x_{j}$ for any $i, j$ (such $x$ exists). It can be verified that the elements in $Z_{G}(x)$ must have the form

$$
g=\left(\begin{array}{cc}
A_{1} & A_{2} \\
A_{3} & A_{4}
\end{array}\right)
$$

where each row and each column of $G$ has only one non-zero entry and $\left(A_{1}\right)_{i j} \neq$ $0 \Leftrightarrow\left(A_{4}\right)_{i j} \neq 0,\left(A_{2}\right)_{i j} \neq 0 \Leftrightarrow\left(A_{3}\right)_{i j} \neq 0$. Hence we see that $Z_{G}^{0}(x)=T$ and $x$ is regular.

(iii) $G$ is the adjoint group of type $D_{n}$. We have the following construction of $G$. Let $V$ be a $2 n$-dimensional vector space equipped with a non-defective quadratic form $Q: V \rightarrow k$. Let $\langle$,$\rangle be the bilinear form associated to Q$ defined by $\langle v, w\rangle=Q(v+w)+Q(v)+Q(w)$. Then $G$ is defined as

$$
\left\{(g, \lambda) \in G L(V) \times k^{*} \mid \forall v \in V, Q(g v)=\lambda Q(v)\right\} /\left\{\left(\mu I, \mu^{2}\right) \mid \mu \in k^{*}\right\} .
$$

Hence the Lie algebra $\mathfrak{g}$ is

$$
\{(x, \lambda) \in \operatorname{End}(V) \times k \mid \forall v \in V,\langle x v, v\rangle=\lambda Q(v)\} /\{(\mu I, 0) \mid \mu \in k\} .
$$

With respect to a suitable basis, we can assume $Q(v)=v^{t} B v$ where

$$
B=\left(\begin{array}{cc}
0 & I_{n \times n} \\
0 & 0
\end{array}\right) \text {. }
$$


A maximal torus of $G$ is $T=\left\{\operatorname{diag}\left(t_{1}, t_{2}, \ldots, t_{n}, \lambda / t_{1}, \lambda / t_{2}, \ldots \lambda / t_{n}\right) \mid t_{i} \in k^{*}, i=\right.$ $\left.1, \ldots, n ; \lambda \in k^{*}\right\} /\left\{\left(\mu I, \mu^{2}\right) \mid \mu \in k^{*}\right\}$. The Lie algebra of $T$ is $\mathfrak{t}=\left\{\operatorname{diag}\left(x_{1}, x_{2}, \ldots\right.\right.$, $\left.\left.x_{n}, \lambda+x_{1}, \lambda+x_{2}, \ldots \lambda+x_{n}\right) \mid x_{i} \in k, i=1, \ldots, n ; \lambda \in k\right\} /\{(\mu I, 0) \mid \mu \in k\}$.

Let $x=\overline{\operatorname{diag}\left(x_{1}, x_{2}, \ldots, x_{n}, x_{1}+\lambda, x_{2}+\lambda, \ldots x_{n}+\lambda\right)} \in \mathfrak{t}$ where $x_{i} \neq x_{j}$, for any $i \neq j$ and $\lambda \neq x_{i}+x_{j}$ for any $i \neq j$ (such $x$ exists), then similarly one can show that $Z_{G}^{0}(x)=T$ and $x$ is regular.

Now denote by $\mathfrak{t}_{0}$ the set of regular elements in $\mathfrak{t}$. From the above construction, one easily sees that $\mathfrak{t}_{0}$ is a dense subset in $\mathfrak{t}$. Thus $\operatorname{dim} \mathfrak{t}_{0}=\operatorname{dim} \mathfrak{t}=r$. Consider the map $\mathfrak{t}_{0} \times G \rightarrow \mathfrak{g},(x, g) \mapsto \operatorname{Ad}(g) x$. The fiber at $y$ in the image is $\{(x, g) \in$ $\left.\mathfrak{t}_{0} \times G \mid \operatorname{Ad}(g) x=y\right\}$. We consider the projection of $\left\{(x, g) \in \mathfrak{t}_{0} \times G \mid \operatorname{Ad}(g) x=y\right\}$ to the first coordinate. The fiber of this projection at $x \in \mathfrak{t}_{0}$ is isomorphic to $Z_{G}(x)$, which has dimension $r$, and the image of this projection is finite. Hence $\operatorname{dim}\left\{(x, g) \in \mathfrak{t}_{0} \times G \mid \operatorname{Ad}(g) x=y\right\}=r$. It follows that the image of the map $\mathfrak{t}_{0} \times G \rightarrow \mathfrak{g},(x, g) \mapsto \operatorname{Ad}(g) x$ has dimension equal to $\operatorname{dim}\left(\mathfrak{t}_{0} \times G\right)-r=\operatorname{dim} \mathfrak{g}$. This proves the lemma.

Remark 6.3. Lemma 6.2 is not always true when $G$ is not adjoint.

6.3. Let $Y$ be the set of semisimple regular elements in $\mathfrak{g}$. By Lemma 6.2, $\operatorname{dim} Y=$ $\operatorname{dim} G$. Let $\widetilde{Y}=\left\{(x, g T) \in Y \times G / T \mid \operatorname{Ad}\left(g^{-1}\right)(x) \in \mathfrak{t}_{0}\right\}$. Define $\pi: \widetilde{Y} \rightarrow Y$ by $\pi(x, g T)=x$. The Weyl group $W=N T / T$ acts (freely) on $\widetilde{Y}$ by $n:(x, g T) \mapsto$ $\left(x, g n^{-1} T\right)$.

Lemma 6.4. $\pi: \widetilde{Y} \rightarrow Y$ is a principal $W$-bundle.

Proof. We show that if $x \in \mathfrak{g}, g, g^{\prime} \in G$ are such that $\operatorname{Ad}\left(g^{-1}\right) x \in \mathfrak{t}_{0}$ and $\operatorname{Ad}\left(g^{\prime-1}\right) x \in$ $\mathfrak{t}_{0}$, then $g^{\prime}=g n^{-1}$ for some $n \in N T$. Let $\operatorname{Ad}\left(g^{-1}\right) x=t_{1} \in \mathfrak{t}_{0}, \operatorname{Ad}\left(g^{\prime-1}\right) x=t_{2} \in \mathfrak{t}_{0}$, then we have $Z_{G}^{0}(x)=Z_{G}^{0}\left(\operatorname{Ad}(g) t_{1}\right)=g Z_{G}^{0}\left(t_{1}\right) g^{-1}=g T g^{-1}$; similarly $Z_{G}^{0}(x)=$ $Z_{G}^{0}\left(\operatorname{Ad}\left(g^{\prime}\right) t_{2}\right)=g^{\prime} Z_{G}^{0}\left(t_{2}\right) g^{\prime-1}=g^{\prime} T g^{\prime-1}$, hence $g^{\prime-1} g \in N T$.

Let $X=\left\{(x, g B) \in \mathfrak{g} \times G / B \mid \operatorname{Ad}\left(g^{-1}\right) x \in \mathfrak{b}\right\}$. Define $\varphi: X \rightarrow \mathfrak{g}$ by $\varphi(x, g B)=$ $x$. The map $\varphi$ is $G$-equivariant with $G$-action on $X$ given by $g_{0}:(x, g B) \mapsto$ $\left(\operatorname{Ad}\left(g_{0}\right) x, g_{0} g B\right)$.

Lemma 6.5. (i) $X$ is an irreducible variety of dimension equal to $\operatorname{dim} G$.

(ii) $\varphi$ is proper and $\varphi(X)=\mathfrak{g}=\bar{Y}$.

(iii) $(x, g T) \rightarrow(x, g B)$ is an isomorphism $\gamma: \tilde{Y} \stackrel{\sim}{\rightarrow} \varphi^{-1}(Y)$.

Proof. (i) and (ii) are easy. For (iii), we only prove that $\gamma$ is a bijection. First we show that $\gamma$ is injective. Suppose $\left(x_{1}, g_{1} T\right),\left(x_{2}, g_{2} T\right) \in \widetilde{Y}$ are such that $\left(x_{1}, g_{1} B\right)=$ $\left(x_{2}, g_{2} B\right)$, then we have $\operatorname{Ad}\left(g_{1}^{-1}\right)\left(x_{1}\right) \in \mathfrak{t}_{0}, \operatorname{Ad}\left(g_{2}^{-1}\right)\left(x_{2}\right) \in \mathfrak{t}_{0}$ and $x_{1}=x_{2}, g_{2}^{-1} g_{1} \in$ $B$. A similar argument as in the proof of Lemma 6.4 shows $g_{2}^{-1} g_{1} \in N T$, hence $g_{2}^{-1} g_{1} \in B \cap N T=T$ and it follows that $g_{1} T=g_{2} T$. Now we show that $\gamma$ is surjective. For $(x, g B) \in \varphi^{-1}(Y)$, we have $x \in Y, \operatorname{Ad}\left(g^{-1}\right)(x) \in \mathfrak{b}$, hence there exists $b \in B, x_{0} \in \mathfrak{t}_{0}$ such that $\operatorname{Ad}\left(g^{-1}\right)(x)=\operatorname{Ad}(b)\left(x_{0}\right)$. Then $\gamma(x, g b T)=(x, g B)$.

Since $\pi: \widetilde{Y} \rightarrow Y$ is a finite covering, $\pi_{!} \overline{\mathbb{Q}}_{l \widetilde{Y}}$ is a well-defined local system on $Y$. Thus the intersection cohomology complex $I C\left(\mathfrak{g}, \pi_{!} \overline{\mathbb{Q}}_{l \widetilde{Y}}\right)$ is well defined.

Proposition 6.6. $\varphi_{!} \overline{\mathbb{Q}}_{l X}$ is canonically isomorphic to $I C\left(\mathfrak{g}, \pi_{!} \overline{\mathbb{Q}}_{l \tilde{Y}}\right)$. Moreover, $\operatorname{End}\left(\varphi_{!} \overline{\mathbb{Q}}_{l X}\right)=\operatorname{End}\left(\pi_{!} \overline{\mathbb{Q}}_{l \tilde{Y}}\right)=\overline{\mathbb{Q}}_{l}[W]$. 
Proof. Note that we have a commutative diagram

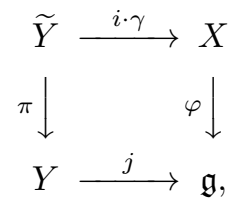

where $i: \varphi^{-1}(Y) \rightarrow X$ and $j$ are inclusions. By base change theorem, we have $\left.\varphi_{!} \overline{\mathbb{Q}}_{l X}\right|_{Y}=\pi_{!} \overline{\mathbb{Q}}_{l \widetilde{Y}}$. Since $\varphi$ is proper and $X$ is smooth of dimension equal to $\operatorname{dim} Y$, we have that the Verdier dual $\mathfrak{D}\left(\varphi_{!} \overline{\mathbb{Q}}_{l X}\right)=\varphi_{!}\left(\mathfrak{D} \overline{\mathbb{Q}}_{l X}\right) \cong \varphi_{!} \overline{\mathbb{Q}}_{l X}[2 \operatorname{dim} Y]$. Hence by the definition of intersection cohomology complex, it is enough to prove that

$$
\forall i>0, \operatorname{dim} \operatorname{supp} \mathcal{H}^{i}\left(\varphi_{!} \overline{\mathbb{Q}}_{l X}\right)<\operatorname{dim} Y-i .
$$

For $x \in \mathfrak{g}$, the stalk $\mathcal{H}_{x}^{i}\left(\varphi_{!} \overline{\mathbb{Q}}_{l X}\right)=H_{c}^{i}\left(\varphi^{-1}(x), \overline{\mathbb{Q}}_{l}\right)$. Hence it is enough to show $\forall i>0, \operatorname{dim}\left\{x \in \mathfrak{g} \mid H_{c}^{i}\left(\varphi^{-1}(x), \overline{\mathbb{Q}}_{l}\right) \neq 0\right\}<\operatorname{dim} Y-i$. If $H_{c}^{i}\left(\varphi^{-1}(x), \overline{\mathbb{Q}}_{l}\right) \neq 0$, then $i \leq 2 \operatorname{dim} \varphi^{-1}(x)$. Hence it is enough to show that

$$
\forall i>0, \operatorname{dim}\left\{x \in \mathfrak{g} \mid \operatorname{dim} \varphi^{-1}(x) \geq i / 2\right\}<\operatorname{dim} Y-i .
$$

Suppose this is not true for some $i$, then $\operatorname{dim}\left\{x \in \mathfrak{g} \mid \operatorname{dim} \varphi^{-1}(x) \geq i / 2\right\} \geq \operatorname{dim} Y-i$. Let $V=\left\{x \in \mathfrak{g} \mid \operatorname{dim} \varphi^{-1}(x) \geq i / 2\right\}$; it is closed in $\mathfrak{g}$ but not equal to $\mathfrak{g}$. Consider the $\operatorname{map} p: Z \rightarrow \mathfrak{g},\left(x, B_{1}, B_{2}\right) \mapsto x$. We have $\operatorname{dim} p^{-1}(V)=\operatorname{dim} V+2 \operatorname{dim} \varphi^{-1}(x) \geq$ $\operatorname{dim} V+i \geq \operatorname{dim} Y$ (for some $x \in V$ ). Thus by Lemma6.1(iii), $p^{-1}(V)$ contains some $Z_{\mathcal{O}}, \mathcal{O}=G$-orbit of $\left(B, n B n^{-1}\right)$ in $\mathcal{B} \times \mathcal{B}$. If $x \in \mathfrak{t}_{0}$, then $\left(x, B, n B n^{-1}\right) \in Z_{\mathcal{O}}$, hence $x$ belongs to the projection of $p^{-1}(V)$ to $\mathfrak{g}$ which has $\operatorname{dimension} \operatorname{dim} V<\operatorname{dim} Y$; but this projection is $G$-invariant hence contains all $Y$. We get a contradiction.

Since $\pi$ is a principal $W$-bundle, we have $\operatorname{End}\left(\pi_{!} \overline{\mathbb{Q}}_{l \widetilde{Y}}\right)=\overline{\mathbb{Q}}_{l}[W]$. It follows that $\operatorname{End}\left(\varphi ! \overline{\mathbb{Q}}_{l X}\right)=\overline{\mathbb{Q}}_{l}[W]$.

6.4. In this subsection, we introduce some sheaves on the variety of semisimple $G$-conjugacy classes in $\mathfrak{g}$ similar to [4, 5].

Let $\mathbf{A}$ be the set of closed $G$-conjugacy classes in $\mathfrak{g}$. These are precisely the semisimple classes in $\mathfrak{g}$ (for a proof in the group case see for example [14, and one can prove the Lie algebra case similarly). By geometric invariant theory, $\mathbf{A}$ has a natural structure of affine variety and there is a well-defined morphism $\sigma: \mathfrak{g} \rightarrow \mathbf{A}$ such that $\sigma(x)$ is the $G$-conjugacy class of $x_{s}$. There is a unique $\varsigma \in \mathbf{A}$ such that $\sigma^{-1}(\varsigma)=\{x \in \mathfrak{g} \mid x$ nilpotent $\}$.

Recall that $Z=\left\{\left(x, B_{1}, B_{2}\right) \in \mathfrak{g} \times \mathcal{B} \times \mathcal{B} \mid x \in \mathfrak{b}_{1} \cap \mathfrak{b}_{2}\right\}$. Define $\tilde{\sigma}: Z \rightarrow \mathbf{A}$ by $\tilde{\sigma}\left(x, B_{1}, B_{2}\right)=\sigma(x)$. For $a \in \mathbf{A}$, let $Z^{a}=\tilde{\sigma}^{-1}(a)$.

Lemma 6.7. We have $\operatorname{dim} Z^{a} \leq d_{0}$, where $d_{0}=\operatorname{dim} G-r$.

Proof. Define $m: Z^{a} \rightarrow \sigma^{-1}(a)$ by $\left(x, B_{1}, B_{2}\right) \mapsto x$. Let $\mathrm{c} \subset \sigma^{-1}(a)$ be a $G$ conjugacy class. Consider $m: m^{-1}(\mathrm{c}) \rightarrow$ c. We have $\operatorname{dim} m^{-1}(\mathrm{c}) \leq \operatorname{dim} \mathrm{c}+$ $2\left(\operatorname{dim} G-r-\operatorname{dim}\right.$ c) $/ 2=\operatorname{dim} G-r$ (use Lemma 6.1(ii)). Since $\sigma^{-1}(a)$ is a union of finitely many $G$-conjugacy classes, it follows that $\operatorname{dim} Z^{a} \leq d_{0}$.

Let $\mathcal{T}=\mathcal{H}^{2 d_{0}} \tilde{\sigma}_{!} \overline{\mathbb{Q}}_{l Z}$. Recall that we set $Z_{\mathcal{O}}=\left\{\left(x, B_{1}, B_{2}\right) \in Z \mid\left(B_{1}, B_{2}\right) \in \mathcal{O}\right\}$, where $\mathcal{O}$ is an orbit of $G$ action on $\mathcal{B} \times \mathcal{B}$. Let $\mathcal{T}^{\mathcal{O}}=\mathcal{H}^{2 d_{0}} \sigma_{!}^{0} \overline{\mathbb{Q}}_{l}$, where $\sigma^{0}: Z_{\mathcal{O}} \rightarrow \mathbf{A}$ is the restriction of $\tilde{\sigma}$ on $Z_{\mathcal{O}}$.

Lemma 6.8. We have $\mathcal{T}^{\mathcal{O}} \cong \bar{\sigma}_{!} \overline{\mathbb{Q}}_{l}$, where $\bar{\sigma}: \mathfrak{t} \rightarrow \boldsymbol{A}$ is the restriction of $\sigma$. 
Proof. The fiber of the natural projection $p r_{23}: Z_{\mathcal{O}} \rightarrow \mathcal{O}$ at $\left(B, n B n^{-1}\right) \in \mathcal{O}$ can be identified with $V=\mathfrak{b} \cap n \mathfrak{b} n^{-1}$. Let $\mathcal{T}^{\mathcal{O}}=\mathcal{H}^{2 d_{0}-2 \operatorname{dim} \mathcal{O}} \sigma_{!}^{\prime} \overline{\mathbb{Q}}_{l}$, where $\sigma^{\prime}: V \rightarrow \mathbf{A}$ is $x \mapsto \sigma(x)$. Let $\mathcal{T}^{\prime \prime \mathcal{O}}=\mathcal{H}^{2 d_{0}+2 \operatorname{dim} H} \sigma_{!}^{\prime \prime} \overline{\mathbb{Q}}_{l}$, where $H=B \cap n B n^{-1}$ and $\sigma^{\prime \prime}$ : $G \times V \rightarrow \mathbf{A}$ is $(g, x) \mapsto \sigma(x)$. Consider the composition $G \times V \stackrel{p r_{2}}{\longrightarrow} V \stackrel{\sigma^{\prime}}{\longrightarrow} \mathbf{A}$ (equal to $\sigma^{\prime \prime}$ ) and the composition $G \times V \stackrel{p}{\rightarrow} H \backslash(G \times V)=Z_{\mathcal{O}} \stackrel{\sigma^{0}}{\rightarrow} \mathbf{A}$ (equal to $\sigma^{\prime \prime}$ ), we obtain

$$
\begin{gathered}
\mathcal{T}^{\prime \prime \mathcal{O}}=\mathcal{H}^{2 d_{0}+2 \operatorname{dim} H}\left(\sigma_{!}^{\prime} p p_{2 !} \overline{\mathbb{Q}}_{l}\right)=\mathcal{H}^{2 d_{0}+2 \operatorname{dim} H}\left(\sigma_{!}^{\prime} \overline{\mathbb{Q}}_{l}[-2 \operatorname{dim} G]\right)=\mathcal{T}^{\mathcal{O}} \\
\mathcal{T}^{\prime \prime \mathcal{O}}=\mathcal{H}^{2 d_{0}+2 \operatorname{dim} H}\left(\sigma_{!}^{0} p ! \overline{\mathbb{Q}}_{l}\right)=\mathcal{H}^{2 d_{0}+2 \operatorname{dim} H}\left(\sigma_{!}^{0} \overline{\mathbb{Q}}_{l}[-2 \operatorname{dim} H]\right)=\mathcal{T}^{\mathcal{O}} .
\end{gathered}
$$

It follows that $\mathcal{T}^{\mathcal{O}}=\mathcal{T}^{\mathcal{O}}$. Now $V$ is fibered over $\mathfrak{t}$ with fibers isomorphic to $\mathfrak{n} \cap n \mathfrak{n} n^{-1}$. The map $\sigma^{\prime}: V \rightarrow \mathbf{A}$ factors through $\bar{\sigma}: \mathfrak{t} \rightarrow \mathbf{A}$. Since $\mathfrak{n} \cap n \mathfrak{n} n^{-1}$ is an affine space of dimension $d_{0}-\operatorname{dim} \mathcal{O}$, we see that $\mathcal{T}^{\mathcal{O}}=\mathcal{T}^{\prime \mathcal{O}} \cong \mathcal{H}^{0} \bar{\sigma}_{!} \overline{\mathbb{Q}}_{l}$. Since $\bar{\sigma}$ is a finite covering (Lemma 6.9), we have $\mathcal{T}^{\mathcal{O}} \cong \bar{\sigma}_{!} \overline{\mathbb{Q}}_{l}$.

Lemma 6.9. The map $\bar{\sigma}: \mathfrak{t} \rightarrow \boldsymbol{A}$ is a finite covering.

Proof. We show that for $x_{1}, x_{2} \in \mathfrak{t}$, if $\sigma\left(x_{1}\right)=\sigma\left(x_{2}\right)$, then there exists $w \in W$ such that $x_{2}=\operatorname{Ad}(w) x_{1}$. Since $\sigma\left(x_{1}\right)=\sigma\left(x_{2}\right)$, there exists $g \in G$ such that $x_{1}=\operatorname{Ad}(g)\left(x_{2}\right)$. It follows that $Z_{G}^{0}\left(x_{1}\right)=g Z_{G}^{0}\left(x_{2}\right) g^{-1}$. We have $T \subset Z_{G}^{0}\left(x_{1}\right)$ and $g T g^{-1} \subset Z_{G}^{0}\left(x_{1}\right)$. Hence there exists $h \in Z_{G}^{0}\left(x_{1}\right)$ such that $h T h^{-1}=g T g^{-1}$. Let $n=g^{-1} h$, we have $x_{2}=\operatorname{Ad}\left(g^{-1}\right) x_{1}=\operatorname{Ad}\left(n h^{-1}\right) x_{1}=\operatorname{Ad}(n) x_{1}$.

Denote $\mathcal{T}_{\varsigma}$ and $\mathcal{T}_{\varsigma}^{\mathcal{O}}$ the stalk of $\mathcal{T}$ and $\mathcal{T}^{\mathcal{O}}$ at $\varsigma$, respectively.

Lemma 6.10. For $w \in W$, let $\mathcal{O}_{w}$ be the $G$-orbit on $\mathcal{B} \times \mathcal{B}$ which contains $\left(B, n_{w} B n_{w}^{-1}\right)$. There is a canonical isomorphism $\mathcal{T}_{\varsigma} \cong \bigoplus_{w \in W} \mathcal{T}_{\varsigma}^{\mathcal{O}_{w}}$.

Proof. We have $\tilde{\sigma}^{-1}(\varsigma)=Z^{\prime}=\left\{\left(x, B_{1}, B_{2}\right) \in \mathfrak{g} \times \mathcal{B} \times \mathcal{B} \mid x \in \mathfrak{n}_{1} \cap \mathfrak{n}_{2}\right\}$. We have a partition $Z^{\prime}=\bigsqcup_{w \in W} Z_{\mathcal{O}_{w}}^{\prime}$, where $Z_{\mathcal{O}_{w}}^{\prime}=\left\{\left(x, B_{1}, B_{2}\right) \in Z^{\prime} \mid\left(B_{1}, B_{2}\right) \in \mathcal{O}_{w}\right\}$. Since $\operatorname{dim} Z^{\prime}=d_{0}$, we have an isomorphism

$$
H_{c}^{2 d_{0}}\left(Z^{\prime}, \overline{\mathbb{Q}}_{l}\right)=\bigoplus_{w \in W} H_{c}^{2 d_{0}}\left(Z_{\mathcal{O}_{w}}^{\prime}, \overline{\mathbb{Q}}_{l}\right)
$$

which is $\mathcal{T}_{\varsigma} \cong \bigoplus_{w \in W} \mathcal{T}_{\varsigma}^{\mathcal{O}_{w}}$.

Recall that we have $\overline{\mathbb{Q}}_{l}[W]=\operatorname{End}\left(\pi_{!} \overline{\mathbb{Q}}_{l \widetilde{Y}}\right)=\operatorname{End}\left(\varphi_{!} \overline{\mathbb{Q}}_{l X}\right)$. In particular, $\varphi_{!} \overline{\mathbb{Q}}_{l X}$ is naturally a $W$-module and $\varphi_{!} \overline{\mathbb{Q}}_{l X} \otimes \varphi ! \overline{\mathbb{Q}}_{l X}$ is naturally a $W$-module (with $W$ acting on the first factor). This induces a $W$-module structure on $\mathcal{H}^{2 d_{0}} \sigma_{!}\left(\varphi_{!} \overline{\mathbb{Q}}_{l X} \otimes\right.$ $\left.\varphi_{!} \overline{\mathbb{Q}}_{l X}\right)=\mathcal{T}$. Hence we obtain a $W$-module structure on the stalk $\mathcal{T}_{\varsigma}$.

Lemma 6.11. Let $w \in W$. Multiplication by $w$ in the $W$-module structure of $\mathcal{T}_{\varsigma}=\bigoplus_{w^{\prime} \in W} \mathcal{T}_{\varsigma}^{\mathcal{O}_{w^{\prime}}}$ defines for any $w^{\prime} \in W$ an isomorphism $\mathcal{T}_{\varsigma}^{\mathcal{O}_{w^{\prime}}} \stackrel{\sim}{\rightarrow} \mathcal{T}_{\varsigma}^{\mathcal{O}_{w w}}$.

Proof. We have an isomorphism

$f: Z_{\mathcal{O}_{w^{\prime}}}^{\prime} \stackrel{\sim}{\rightarrow} Z_{\mathcal{O}_{w w^{\prime}}}^{\prime},\left(x, g B g^{-1}, g n_{w^{\prime}} B n_{w^{\prime}}^{-1} g^{-1}\right) \mapsto\left(x, g n_{w}^{-1} B n_{w} g^{-1}, g n_{w^{\prime}} B n_{w^{\prime}}^{-1} g^{-1}\right)$.

This induces an isomorphism

$$
H_{c}^{2 d_{0}}\left(Z_{\mathcal{O}_{w^{\prime}}}^{\prime}, \overline{\mathbb{Q}}_{l}\right) \stackrel{\sim}{\rightarrow} H_{c}^{2 d_{0}}\left(Z_{\mathcal{O}_{w w^{\prime}}}^{\prime}, \overline{\mathbb{Q}}_{l}\right),
$$

which is just multiplication by $w$. 
6.5. Let $\hat{W}$ be the set of simple modules (up to isomorphism) for the Weyl group $W$ of $G$ (A description of $\hat{W}$ is given for example in [7). Given a semisimple object $M$ of some abelian category such that $M$ is a $W$-module, we write $M_{\rho}=$ $\operatorname{Hom}_{\overline{\mathbb{Q}}_{l}[W]}(\rho, M)$ for $\rho \in \hat{W}$. We have $M=\bigoplus_{\rho \in \hat{W}}\left(\rho \otimes M_{\rho}\right)$ with $W$ acting on the $\rho$-factor and $M_{\rho}$ is in our abelian category. In particular, we have

$$
\pi_{!} \overline{\mathbb{Q}}_{l \widetilde{Y}}=\bigoplus_{\rho \in \hat{W}}\left(\rho \otimes\left(\pi_{!} \overline{\mathbb{Q}}_{l \widetilde{Y}}\right)_{\rho}\right),
$$

where $\left(\pi_{!} \overline{\mathbb{Q}}_{l \tilde{Y}}\right)_{\rho}$ is an irreducible local system on $Y$. We have

$$
\varphi_{!} \overline{\mathbb{Q}}_{l X}=\bigoplus_{\rho \in \hat{W}}\left(\rho \otimes\left(\varphi_{!} \overline{\mathbb{Q}}_{l X}\right)_{\rho}\right),
$$

where $\left(\varphi ! \overline{\mathbb{Q}}_{l X}\right)_{\rho}=I C\left(\bar{Y},\left(\pi_{!} \overline{\mathbb{Q}}_{l \tilde{Y}}\right)_{\rho}\right)$. Moreover, for $a \in \mathbf{A}$, we have $\mathcal{T}_{a}=\bigoplus_{\rho \in \hat{W}}(\rho \otimes$ $\left.\left(\mathcal{T}_{a}\right)_{\rho}\right)$. Set

$$
\bar{Y}^{\varsigma}=\{x \in \bar{Y} \mid \sigma(x)=\varsigma\}, X^{\varsigma}=\varphi^{-1}\left(\bar{Y}^{\varsigma}\right) \subset X .
$$

We have $\bar{Y}^{\varsigma}=\{x \in \mathfrak{g} \mid x$ nilpotent $\}$. Let $\varphi^{\varsigma}: X^{\varsigma} \rightarrow \bar{Y}^{\varsigma}$ be the restriction of $\varphi: X \rightarrow \mathfrak{g}$.

Lemma 6.12. (i) $X^{\varsigma}$ and $\bar{Y}^{\varsigma}$ are irreducible varieties of dimension $d_{0}=\operatorname{dim} G-r$.

(ii) We have $\left.\left(\varphi_{!} \overline{\mathbb{Q}}_{l X}\right)\right|_{\bar{Y}^{\varsigma}}=\varphi_{!}^{\varsigma} \overline{\mathbb{Q}}_{l X^{\varsigma}}$. Moreover, $\varphi_{!}^{\varsigma} \overline{\mathbb{Q}}_{l X^{\varsigma}}\left[d_{0}\right]$ is a semisimple perverse sheaf on $\bar{Y}^{\varsigma}$.

(iii) We have $\left.\left(\varphi_{!} \overline{\mathbb{Q}}_{l X}\right)_{\rho}\right|_{\bar{Y}_{\varsigma}} \neq 0$ for any $\rho \in \hat{W}$.

Proof. (i) $\bar{Y}^{\varsigma}$, which is the nilpotent variety, is well-known to be irreducible of dimension $\operatorname{dim} G-r$. We have $X^{\varsigma}=\left\{(x, g B) \in \mathfrak{g} \times \mathcal{B} \mid \operatorname{Ad}\left(g^{-1}\right)(x) \in \mathfrak{n}\right\}$. By projection to the second coordinate, we see that $\operatorname{dim} X^{\varsigma}=\operatorname{dim} \mathfrak{n}+\operatorname{dim} \mathcal{B}=\operatorname{dim} G-$ $r$. This proves (i).

The first assertion of (ii) follows by applying base change theorem to the following commutative diagram:

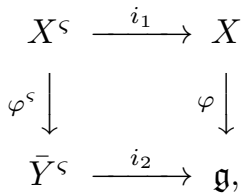

where $i_{1}, i_{2}$ are inclusions. Since $\varphi^{\varsigma}$ is proper, by similar argument as in the proof of Proposition 6.6 to show that $\varphi_{!}^{\varsigma} \overline{\mathbb{Q}}_{l X^{\varsigma}}\left[d_{0}\right]$ is a perverse sheaf, it suffices to show

$$
\forall i \geq 0, \operatorname{dim} \operatorname{supp} \mathcal{H}^{i}\left(\varphi_{!}^{\varsigma} \overline{\mathbb{Q}}_{l X \varsigma}\right) \leq \operatorname{dim} \bar{Y}^{\varsigma}-i .
$$

It is enough to show $\forall i \geq 0, \operatorname{dim}\left\{x \in \bar{Y}^{\varsigma} \mid \operatorname{dim}\left(\varphi^{\varsigma}\right)^{-1}(x) \geq i / 2\right\} \leq \operatorname{dim} \bar{Y}^{\varsigma}-i$. If this is not true for some $i \geq 0$, it would follow that the variety $\left\{\left(x, B_{1}, B_{2}\right) \in\right.$ $\left.\mathfrak{g} \times \mathcal{B} \times \mathcal{B} \mid x \in \mathfrak{n}_{1} \cap \mathfrak{n}_{2}\right\}$ has dimension greater than $\operatorname{dim} \bar{Y}^{\varsigma}=\operatorname{dim} G-r$, which contradicts Lemma 6.1. This proves that $\varphi_{!}^{\varsigma} \overline{\mathbb{Q}}_{l X^{\varsigma}}\left[d_{0}\right]$ is a perverse sheaf. It is semisimple by the decomposition theorem [1]. This proves (ii).

Now we prove (iii). By Lemma 6.8, we have $\mathcal{T}_{\varsigma}^{\mathcal{O}_{1}}=H_{c}^{0}\left(\mathfrak{t} \cap \sigma^{-1}(\varsigma), \overline{\mathbb{Q}}_{l}\right) \neq 0$. From Lemma 6.11, we see that the $W$-module structure defines an injective map $\overline{\mathbb{Q}}_{l}[W] \otimes \mathcal{T}_{\varsigma}^{\mathcal{O}_{1}} \rightarrow \mathcal{T}_{\varsigma}$. Since $\mathcal{T}_{\varsigma}^{\mathcal{O}_{1}} \neq 0$, we have $\left(\overline{\mathbb{Q}}_{l}[W] \otimes \mathcal{T}_{\varsigma}^{\mathcal{O}_{1}}\right)_{\rho} \neq 0$ for any $\rho \in \hat{W}$, hence $\left(\mathcal{T}_{\varsigma}\right)_{\rho} \neq 0$. We have $\mathcal{T}_{\varsigma}=H_{c}^{2 d_{0}}\left(\bar{Y}^{\varsigma}, \varphi ! \overline{\mathbb{Q}}_{l X} \otimes \varphi_{!} \overline{\mathbb{Q}}_{l X}\right)$, hence

$$
\bigoplus_{\rho \in \hat{W}} \rho \otimes\left(\mathcal{T}_{\varsigma}\right)_{\rho}=\bigoplus_{\rho \in \hat{W}} \rho \otimes H_{c}^{2 d_{0}}\left(\bar{Y}^{\varsigma},\left(\varphi ! \overline{\mathbb{Q}}_{l X}\right)_{\rho} \otimes \varphi ! \overline{\mathbb{Q}}_{l X}\right)
$$


This implies that $\left(\mathcal{T}_{\varsigma}\right)_{\rho}=H_{c}^{2 d_{0}}\left(\bar{Y}^{\varsigma},\left(\varphi ! \overline{\mathbb{Q}}_{l X}\right)_{\rho} \otimes \varphi_{!} \overline{\mathbb{Q}}_{l X}\right)$. Thus it follows from $\left(\mathcal{T}_{\varsigma}\right)_{\rho} \neq$ 0 that $\left.\left(\varphi_{!} \overline{\mathbb{Q}}_{l X}\right)_{\rho}\right|_{\bar{Y} \varsigma} \neq 0$ for any $\rho \in \hat{W}$.

Let $\mathfrak{A}$ be the set of all pairs $(\mathrm{c}, \mathcal{F})$ where c is a nilpotent $G$-conjugacy class in $\mathfrak{g}$ and $\mathcal{F}$ is an irreducible $G$-equivariant local system on c (up to isomorphism).

Proposition 6.13. (i) The restriction map $\operatorname{End}_{\mathcal{D}(\bar{Y})}\left(\varphi_{!} \overline{\mathbb{Q}}_{l X}\right) \rightarrow \operatorname{End}_{\mathcal{D}\left(\bar{Y}^{\varsigma}\right)}\left(\varphi_{!}^{\varsigma} \overline{\mathbb{Q}}_{l X^{\varsigma}}\right)$ is an isomorphism.

(ii) For any $\rho \in \hat{W}$, there is a unique $(\mathrm{c}, \mathcal{F}) \in \mathfrak{A}$ such that $\left.\left(\varphi_{!} \overline{\mathbb{Q}}_{l X}\right)_{\rho}\right|_{\bar{Y}^{\varsigma}}\left[d_{0}\right]$ is $\operatorname{IC}(\overline{\mathrm{c}}, \mathcal{F})[\operatorname{dim} \mathrm{c}]$ regarded as a simple perverse sheaf on $\bar{Y}^{\varsigma}$ (zero outside $\overline{\mathrm{c}}$ ). Moreover, $\rho \mapsto(\mathrm{c}, \mathcal{F})$ is an injective map $\gamma: \hat{W} \rightarrow \mathfrak{A}$.

Proof. (i). Recall that we have $\varphi ! \overline{\mathbb{Q}}_{l X}=\bigoplus_{\rho \in \hat{W}} \rho \otimes\left(\varphi_{!} \overline{\mathbb{Q}}_{l X}\right)_{\rho}$ where $\left(\varphi_{!} \overline{\mathbb{Q}}_{l X}\right)_{\rho}[\operatorname{dim} \bar{Y}]$ are simple perverse sheaves on $\bar{Y}$. Thus we have $\left.\varphi_{!} \overline{\mathbb{Q}}_{l X}\right|_{\bar{Y}^{\varsigma}}=\varphi_{!}^{\varsigma} \overline{\mathbb{Q}}_{l X^{\varsigma}}=\bigoplus_{\rho \in \hat{W}} \rho \otimes$ $\left.\left(\varphi ! \overline{\mathbb{Q}}_{l X}\right)_{\rho}\right|_{\bar{Y}^{\varsigma}}$ (we use Lemma 6.12(ii)). The restriction map $\operatorname{End}_{\mathcal{D}(\bar{Y})}\left(\varphi_{!} \overline{\mathbb{Q}}_{l X}\right) \rightarrow$ $\operatorname{End}_{\mathcal{D}\left(\bar{Y}^{\varsigma}\right)}\left(\varphi_{!}^{\varsigma} \overline{\mathbb{Q}}_{l X^{\varsigma}}\right)$ is factorized as

$\bigoplus_{\rho \in \hat{W}} \operatorname{End}_{\mathcal{D}(\bar{Y})}\left(\rho \otimes\left(\varphi_{!} \overline{\mathbb{Q}}_{l X}\right)_{\rho}\right) \stackrel{b}{\rightarrow} \bigoplus_{\rho \in \hat{W}} \operatorname{End}_{\mathcal{D}\left(\bar{Y}^{\varsigma}\right)}\left(\left.\rho \otimes\left(\varphi_{!} \overline{\mathbb{Q}}_{l X}\right)_{\rho}\right|_{\bar{Y}^{\varsigma}}\right) \stackrel{c}{\rightarrow} \operatorname{End}_{\mathcal{D}\left(\bar{Y}^{\varsigma}\right)}\left(\varphi_{!}^{\varsigma} \overline{\mathbb{Q}}_{l X^{\varsigma}}\right)$

where

$b=\bigoplus_{\rho} b_{\rho}, b_{\rho}: \operatorname{End}(\rho) \otimes \operatorname{End}_{\mathcal{D}(\bar{Y})}\left(\left(\varphi_{!} \overline{\mathbb{Q}}_{l X}\right)_{\rho}\right) \rightarrow \operatorname{End}(\rho) \otimes \operatorname{End}_{\mathcal{D}\left(\bar{Y}^{\varsigma}\right)}\left(\left.\left(\varphi_{!} \overline{\mathbb{Q}}_{l X}\right)_{\rho}\right|_{\bar{Y}^{\varsigma}}\right)$.

By Lemma 6.12(iii), $\left.\left(\varphi_{!} \overline{\mathbb{Q}}_{l X}\right)_{\rho}\right|_{\bar{Y} \varsigma} \neq 0$, thus

$$
\operatorname{End}_{\mathcal{D}(\bar{Y})}\left(\left(\varphi_{!} \overline{\mathbb{Q}}_{l X}\right)_{\rho}\right)=\overline{\mathbb{Q}}_{l} \subset \operatorname{End}_{\mathcal{D}\left(\bar{Y}^{\varsigma}\right)}\left(\left.\left(\varphi_{!} \overline{\mathbb{Q}}_{l X}\right)_{\rho}\right|_{\bar{Y}^{\varsigma}}\right) .
$$

It follows that $b_{\rho}$ and thus $b$ are injective. Since $c$ is also injective, the restriction map in injective. Hence it remains to show that

$$
\operatorname{dim} \operatorname{End}_{\mathcal{D}\left(\bar{Y}^{\varsigma}\right)}\left(\varphi_{!}^{\varsigma} \overline{\mathbb{Q}}_{l X^{\varsigma}}\right)=\operatorname{dim} \operatorname{End}_{\mathcal{D}(\bar{Y})}\left(\varphi_{!} \overline{\mathbb{Q}}_{l X}\right) .
$$

For $A, A^{\prime}$ two simple perverse sheaves on a variety $X$, we have $H_{c}^{0}\left(X, A \otimes A^{\prime}\right)=0$ if and only if $A$ is not isomorphic to $\mathfrak{D}\left(A^{\prime}\right)$ and $\operatorname{dim} H_{c}^{0}(X, A \otimes \mathfrak{D}(A))=1$ (see [6], Section 7.4). We apply this to the semisimple perverse sheaf $\varphi_{!}^{\varsigma} \overline{\mathbb{Q}}_{l X^{\varsigma}}\left[d_{0}\right]$ on $\bar{Y}^{\varsigma}$ and get $\operatorname{dim} \operatorname{End}_{\mathcal{D}\left(\bar{Y}^{\varsigma}\right)}\left(\varphi_{!}^{\varsigma} \overline{\mathbb{Q}}_{l X^{\varsigma}}\right)=\operatorname{dim} H_{c}^{0}\left(\bar{Y}^{\varsigma}, \varphi_{!}^{\varsigma} \overline{\mathbb{Q}}_{l X^{\varsigma}}\left[d_{0}\right] \otimes \mathfrak{D}\left(\varphi_{!}^{\varsigma} \overline{\mathbb{Q}}_{l X^{\varsigma}}\left[d_{0}\right]\right)\right)$. We have

$$
\begin{aligned}
& \operatorname{dim} H_{c}^{0}\left(\bar{Y}^{\varsigma}, \varphi_{!}^{\varsigma} \overline{\mathbb{Q}}_{l X^{\varsigma}}\left[d_{0}\right] \otimes \mathfrak{D}\left(\varphi_{!}^{\varsigma} \overline{\mathbb{Q}}_{l X^{\varsigma}}\left[d_{0}\right]\right)\right)=\operatorname{dim} H_{c}^{0}\left(\bar{Y}^{\varsigma}, \varphi_{!}^{\varsigma} \overline{\mathbb{Q}}_{l X^{\varsigma}}\left[d_{0}\right] \otimes \varphi_{!}^{\varsigma} \overline{\mathbb{Q}}_{l X^{\varsigma}}\left[d_{0}\right]\right) \\
& =\operatorname{dim} H_{c}^{2 d_{0}}\left(\bar{Y}^{\varsigma}, \varphi_{!}^{\varsigma} \overline{\mathbb{Q}}_{l X^{\varsigma}} \otimes \varphi_{!}^{\varsigma} \overline{\mathbb{Q}}_{l X^{\varsigma}}\right)=\operatorname{dim} H_{c}^{2 d_{0}}\left(\bar{Y}^{\varsigma}, \varphi_{!} \overline{\mathbb{Q}}_{l X} \otimes \varphi_{!} \overline{\mathbb{Q}}_{l X}\right) \\
& =\operatorname{dim} \mathcal{T}_{\varsigma}=\sum_{w \in W} \operatorname{dim} \mathcal{T}_{\varsigma}^{\mathcal{O}_{w}} \text {. }
\end{aligned}
$$

(The third equality follows from Lemma 6.12(ii) and the last one follows from Lemma 6.10.)

We have $\mathcal{T}_{\varsigma}^{\mathcal{O}_{w}}=H_{c}^{0}\left(\bar{\sigma}^{-1}(\varsigma), \overline{\mathbb{Q}}_{l}\right)$ (see Lemma 6.8), hence $\operatorname{dim} \mathcal{T}_{\varsigma}^{\mathcal{O}_{w}}=1$ and

$$
\sum_{w \in W} \operatorname{dim} \mathcal{T}_{\varsigma}^{\mathcal{O}_{w}}=|W|=\operatorname{dim} \operatorname{End}_{\mathcal{D}(\bar{Y})}\left(\varphi ! \overline{\mathbb{Q}}_{l X}\right) .
$$

Thus (i) is proved.

From the proof of (i) we see that both $b$ and $c$ are isomorphisms. It follows that the perverse sheaf $\left.\left(\varphi_{!} \overline{\mathbb{Q}}_{l X}\right)_{\rho}\right|_{\bar{Y}^{\varsigma}}\left[d_{0}\right]$ on $\bar{Y}^{\varsigma}$ is simple and that for $\rho, \rho^{\prime} \in \hat{W}$, we have $\left.\left.\left(\varphi ! \overline{\mathbb{Q}}_{l X}\right)_{\rho}\right|_{\bar{Y}^{\varsigma}}\left[d_{0}\right] \cong\left(\varphi_{!} \overline{\mathbb{Q}}_{l X}\right)_{\rho^{\prime}}\right|_{\bar{Y}^{\varsigma}}\left[d_{0}\right]$ if and only if $\rho=\rho^{\prime}$. Since the simple perverse 
sheaf $\left.\left(\varphi_{!} \overline{\mathbb{Q}}_{l X}\right)_{\rho}\right|_{\bar{Y}^{\varsigma}}\left[d_{0}\right]$ is $G$-equivariant and $\bar{Y}^{\varsigma}$ consists of finitely many nilpotent $G$-conjugacy classes, $\left.\left(\varphi_{!} \overline{\mathbb{Q}}_{l X}\right)_{\rho}\right|_{\bar{Y}^{\varsigma}}\left[d_{0}\right]$ must be as in (ii).

6.6. In this subsection let $G=S O_{N}(\mathbf{k})$ (resp. $S p_{2 n}(\mathbf{k})$ ) and $\mathfrak{g}=\mathfrak{o}_{N}(\mathbf{k}$ ) (resp. $\left.\mathfrak{s p}_{2 n}(\mathbf{k})\right)$ the Lie algebra of $G$. Let $G_{a d}$ be an adjoint group over $\mathbf{k}$ of the same type as $G$ and let $\mathfrak{g}_{a d}$ be the Lie algebra of $G_{a d}$. For $q$ a power of 2, let $G\left(\mathbf{F}_{q}\right), \mathfrak{g}\left(\mathbf{F}_{q}\right)$ be the fixed points of a split Frobenius map $\mathfrak{F}_{q}$ relative to $\mathbf{F}_{q}$ on $G$, g. Let $G_{a d}\left(\mathbf{F}_{q}\right)$, $\mathfrak{g}_{a d}\left(\mathbf{F}_{q}\right)$ be defined as $G\left(\mathbf{F}_{q}\right), \mathfrak{g}\left(\mathbf{F}_{q}\right)$. Let $\mathfrak{A}$ be the set of all pairs $(\mathrm{c}, \mathcal{F})$ where c is a nilpotent $G$-orbit in $\mathfrak{g}$ and $\mathcal{F}$ is an irreducible $G$-equivariant local system on c (up to isomorphism). Let $\mathfrak{A}_{a d}$ be defined for $G_{a d}$ as in the introduction. We show that the number of elements in $\mathfrak{A}_{a d}$ is equal to the number of elements in $\mathfrak{A}$.

We first show that the number of elements in $\mathfrak{A}$ is equal to the number of nilpotent $G\left(\mathbf{F}_{q}\right)$-orbits in $\mathfrak{g}\left(\mathbf{F}_{q}\right)$ (for $q$ large). To see this we can assume $\mathbf{k}=\overline{\mathbf{F}}_{2}$. Pick representatives $x_{1}, \ldots, x_{M}$ for the nilpotent $G$-orbits in $\mathfrak{g}$. If $q$ is large enough, the Frobenius map $\mathfrak{F}_{q}$ keeps $x_{i}$ fixed and acts trivially on $Z_{G}\left(x_{i}\right) / Z_{G}^{0}\left(x_{i}\right)$. Then the number of $G\left(\mathbf{F}_{q}\right)$-orbits in the $G$-orbit of $x_{i}$ is equal to the number of irreducible representations of $Z_{G}\left(x_{i}\right) / Z_{G}^{0}\left(x_{i}\right)$ hence to the number of $G$-equivariant irreducible local systems on the $G$-orbit of $x_{i}$. Similarly, the number of elements in $\mathfrak{A}_{a d}$ is equal to the number of nilpotent $G_{a d}\left(\mathbf{F}_{q}\right)$-orbits in $\mathfrak{g}_{a d}\left(\mathbf{F}_{q}\right)$.

On the other hand, the number of nilpotent $G\left(\mathbf{F}_{q}\right)$-orbits in $\mathfrak{g}\left(\mathbf{F}_{q}\right)$ is equal to the number of nilpotent $G_{a d}\left(\mathbf{F}_{q}\right)$-orbits in $\mathfrak{g}_{a d}\left(\mathbf{F}_{q}\right)$. In fact, we have a morphism $G \rightarrow$ $G_{a d}$ which is an isomorphism of abstract groups and an obvious bijective morphism $\mathcal{U} \rightarrow \mathcal{U}_{a d}$ between the nilpotent variety $\mathcal{U}$ of $\mathfrak{g}$ and the nilpotent variety $\mathcal{U}_{a d}$ of $\mathfrak{g}_{a d}$. Thus the nilpotent orbits in $\mathfrak{g}$ and $\mathfrak{g}_{a d}$ are in bijection and the corresponding component groups of centralizers are isomorphic. It follows that $|\mathfrak{A}|=\left|\mathfrak{A}_{a d}\right|$.

Corollary 6.14. $|\mathfrak{A}|=\left|\mathfrak{A}_{a d}\right|=|\hat{W}|$.

Proof. Assume $G=S O_{N}(\mathbf{k})$. From Proposition 5.1 (i), Corollary 5.2 and the above argument we see that $|\mathfrak{A}|=\left|\mathfrak{A}_{a d}\right| \leq|\hat{W}|$. On the other hand, by Proposition 6.13(ii), we have $\left|\mathfrak{A}_{a d}\right| \geq|\hat{W}|$.

Assume $G=S p_{2 n}(\mathbf{k})$. It is known in [9] that the number of nilpotent $G\left(\mathbf{F}_{q}\right)$ orbits in $\mathfrak{g}\left(\mathbf{F}_{q}\right)$ is equal to $|\hat{W}|$. The assertion follows from the above argument.

Theorem 6.15. The map $\gamma$ in Proposition 6.13(ii) is a bijection.

Corollary 6.16. Proposition 4.1, Corollary 4.2, Proposition 5.1, Corollary 5.2 hold with all "at most" removed.

Proof. For $q$ large enough, this follows from Corollary 6.14. Now let $q$ be an arbitrary power of 2 . The Frobenius map $\mathfrak{F}_{q}$ acts trivially on $W$. Since we have the Springer correspondence map $\gamma$ in Proposition 6.13(ii), for each pair $(\mathrm{c}, \mathcal{F}) \in \mathfrak{A}_{a d}$, c is stable under the Frobenius map $\mathfrak{F}_{q}$ and we have $\mathfrak{F}_{q}^{-1}(\mathcal{F}) \cong \mathcal{F}$. Pick a rational point $x$ in c. The $G_{a d}$-equivariant local systems on c are in oneto-one correspondence with the isomorphism classes of the irreducible representations of $Z_{G_{a d}}(x) / Z_{G_{a d}}^{0}(x)$. Since $Z_{G_{a d}}(x) / Z_{G_{a d}}^{0}(x)$ is abelian (see Proposition 7.1) and the Frobenius map $\mathfrak{F}_{q}$ acts trivially on the irreducible representations of $Z_{G_{a d}}(x) / Z_{G_{a d}}^{0}(x), \mathfrak{F}_{q}$ acts trivially on $Z_{G_{a d}}(x) / Z_{G_{a d}}^{0}(x)$. Thus it follows that the number of nilpotent $G_{a d}\left(\mathbf{F}_{q}\right)$-orbits in $\mathfrak{g}_{a d}\left(\mathbf{F}_{q}\right)$ is independent of $q$ hence it is equal to $\left|\mathfrak{A}_{a d}\right|=|\hat{W}|$. 
A corollary of Theorem 6.15 is that in this case there are no cuspidal local systems similarly defined as in [4. This result does not extend to exceptional Lie algebras. (In type $F_{4}$, characteristic 2 , the results of [11] suggest that a cuspidal local system exists on a nilpotent class.)

\section{Component groups of Centralizers}

In this section we describe the component groups of centralizers $Z_{G}(x) / Z_{G}^{0}(x)$, where $G=S O_{N}(\mathbf{k})$ and $x \in \mathfrak{o}_{N}(\mathbf{k})$ is nilpotent.

Proposition 7.1. (i) Assume $V$ is defective. Let $x \in \mathfrak{o}(V)$ be a nilpotent element corresponding to the form module $\left(\lambda_{1}\right)_{\chi\left(\lambda_{1}\right)}^{m_{1}}\left(\lambda_{2}\right)_{\chi\left(\lambda_{2}\right)}^{m_{2}} \cdots\left(\lambda_{s}\right)_{\chi\left(\lambda_{s}\right)}^{m_{s}}$. Denote by $n_{1}$ the cardinality of $\left\{1 \leq i \leq s-1 \mid \chi\left(\lambda_{i}\right)+\chi\left(\lambda_{i+1}\right) \leq \lambda_{i}, \chi\left(\lambda_{i}\right) \neq \lambda_{i} / 2\right\}$. We have $Z_{O(V)}(x) / Z_{O(V)}^{0}(x)=\left(\mathbb{Z}_{2}\right)^{n_{1}}$.

(ii) Assume $V$ is non-defective. Let $x \in \mathfrak{o}(V)$ be a nilpotent element corresponding to the form module $\left(\lambda_{1}\right)_{\chi\left(\lambda_{1}\right)}^{m_{1}}\left(\lambda_{2}\right)_{\chi\left(\lambda_{2}\right)}^{m_{2}} \cdots\left(\lambda_{s}\right)_{\chi\left(\lambda_{s}\right)}^{m_{s}}$. Denote by $n_{2}$ the cardinality of $\left\{1 \leq i \leq s \mid \chi\left(\lambda_{i}\right)+\chi\left(\lambda_{i+1}\right) \leq \lambda_{i}, \quad \chi\left(\lambda_{i}\right) \neq \lambda_{i} / 2\right\}$ (here define $\left.\chi\left(\lambda_{s+1}\right)=0\right)$. Assume $n_{2} \geq 1$. Then $Z_{S O(V)}(x) / Z_{S O(V)}^{0}(x)=\left(\mathbb{Z}_{2}\right)^{n_{2}-1}$.

Proof. (i) We write $Z=Z_{O(V)}(x)$ and $Z^{0}=Z_{O(V)}^{0}(x)$ for simplicity. We can assume that $q$ is large enough. (i) is proved in two steps.

Step 1: We show that $Z / Z^{0}$ is an abelian group of order $2^{n_{1}}$.

The group $Z / Z^{0}$ has $2^{n_{1}}$ conjugacy classes, since the $G$-orbit of $x$ splits into $2^{n_{1}}$ $G\left(\mathbf{F}_{q}\right)$-orbits in $\mathfrak{g}\left(\mathbf{F}_{q}\right)$ (Corollary [6.16). We show $\left|Z / Z^{0}\right|=2^{n_{1}}$ by showing that

$$
\left|Z\left(\mathbf{F}_{q}\right)\right|=2^{n_{1}} q^{\operatorname{dim}(Z)}+\text { lower terms in } q .
$$

We prove $(*)$ by induction on $n_{1}$. Suppose $n_{1}=0$, then $Z / Z^{0}$ has only one conjugacy class. It follows that $Z / Z^{0}=\{1\}$ and $(*)$ holds for $n_{1}=0$. Suppose $n_{1} \geq 1$. Let $t$ be the minimal integer such that $\chi\left(\lambda_{t}\right) \neq \lambda_{t} / 2$ and $\chi\left(\lambda_{t}\right)+\chi\left(\lambda_{t+1}\right) \leq \lambda_{t}$. Let $V_{1}=\left(\lambda_{1}\right)_{\chi\left(\lambda_{1}\right)}^{m_{1}}\left(\lambda_{2}\right)_{\chi\left(\lambda_{2}\right)}^{m_{2}} \cdots\left(\lambda_{t}\right)_{\chi\left(\lambda_{t}\right)}^{m_{t}}$ and $V_{2}=\left(\lambda_{t+1}\right)_{\chi\left(\lambda_{t+1}\right)}^{m_{t+1}} \cdots\left(\lambda_{s}\right)_{\chi\left(\lambda_{s}\right)}^{m_{s}}$. Then $V_{1}$ is non-defective. Write $Z_{i}=Z_{O\left(V_{i}\right)}\left(V_{i}\right)$ and $Z_{i}^{0}=Z_{O\left(V_{i}\right)}^{0}\left(V_{i}\right), i=1,2$. We have $\left|Z_{S O\left(V_{1}\right)}\left(V_{1}\right) / Z_{S O\left(V_{1}\right)}^{0}\left(V_{1}\right)\right|=1 \Rightarrow\left|Z_{1} / Z_{1}^{0}\right|=2$. It follows that $\left|Z_{1}\left(\mathbf{F}_{q}\right)\right|=2 q^{\operatorname{dim} Z_{1}}+$ lower terms in $q$. We show that $\left|Z\left(\mathbf{F}_{q}\right)\right|=\left|Z_{1}\left(\mathbf{F}_{q}\right)\right| \cdot\left|Z_{2}\left(\mathbf{F}_{q}\right)\right| \cdot q^{\operatorname{dim} \operatorname{Hom}_{A}\left(V_{1}, V_{2}\right)}$. Then the assertion $(*)$ follows from induction hypothesis since we have $\operatorname{dim} Z_{1}+\operatorname{dim} Z_{2}+$ $\operatorname{dim} \operatorname{Hom}_{A}\left(V_{1}, V_{2}\right)=\operatorname{dim} Z$.

Consider $V_{1}$ as an element in the Grassmannian variety $G r(V, r)$ of dimension $r=\sum_{j=1}^{t} m_{j} \lambda_{j}$. Then $C(V)=\{g \in G L(V) \mid g x=x g\}$ acts on $G r(V, r)$. We have $C(V)\left(V_{1} \oplus V_{2}\right)=C(V) V_{1} \oplus C(V) V_{2} \cong V_{1} \oplus V_{2}$. By our choice of $V_{1}$ and $V_{2}$, it follows that $C(V) V_{1} \cong V_{1}$ and $C(V) V_{2} \cong V_{2}$. Thus the orbit of $V_{1}$ under $C(V)$ coincides with the orbit of $V_{1}$ under the action of $Z$. It is easy to verify that this orbit consists of $q^{\operatorname{dim} \operatorname{Hom}_{A}\left(V_{1}, V_{2}\right)}$ elements (using $C(V)$ action). Since the stabilizer of $V_{1}$ in $Z$ is the product of $Z_{1}$ and $Z_{2}$, we get $\left|Z\left(\mathbf{F}_{q}\right)\right|=\left|Z_{1}\left(\mathbf{F}_{q}\right)\right| \cdot\left|Z_{2}\left(\mathbf{F}_{q}\right)\right| \cdot q^{\operatorname{dim} \operatorname{Hom}_{A}\left(V_{1}, V_{2}\right)}$.

Step 2: We show that there is a subgroup $\left(\mathbb{Z}_{2}\right)^{n_{1}} \subset Z / Z^{0}$. Thus $Z / Z^{0}$ has to be $\left(\mathbb{Z}_{2}\right)^{n_{1}}$. Let $1 \leq i_{1}, \ldots, i_{n_{1}} \leq s-1$ be such that $\chi\left(\lambda_{i_{j}}\right)>\lambda_{i_{j}} / 2$ and $\chi\left(\lambda_{i_{j}}\right)+$ $\chi\left(\lambda_{i_{j}+1}\right) \leq \lambda_{i_{j}}, j=1, \ldots, n_{1}$. Let $V_{j}=\left(\lambda_{i_{j-1}+1}\right)_{\chi\left(\lambda_{i_{j-1}+1}\right)}^{m_{i_{i_{j}}+1}} \cdots\left(\lambda_{i_{j}}\right)_{\chi\left(\lambda_{i_{j}}\right)}^{m_{i_{j}}}, j=$ $1, \ldots, n_{1}+1$, where $i_{0}=0, i_{n_{1}+1}=s$. Then $V=V_{1} \oplus V_{2} \oplus \cdots \oplus V_{n_{1}+1}$, where $V_{i}$, $i=1, \ldots, n_{1}$ are non-defective and $V_{n_{1}+1}$ is defective and non-degenerate. We have $Z_{O\left(V_{i}\right)}\left(V_{i}\right) / Z_{O\left(V_{i}\right)}^{0}\left(V_{i}\right)=\mathbb{Z}_{2}, i=1, \ldots, n_{1}$, and $Z_{O\left(V_{n_{1}+1}\right)}\left(V_{n_{1}+1}\right) / Z_{O\left(V_{n_{1}+1}\right)}^{0}\left(V_{n_{1}+1}\right)$ 
$=\{1\}$. Take $g_{i} \in Z_{O\left(V_{i}\right)}\left(V_{i}\right)$ such that $g_{i} Z_{O\left(V_{i}\right)}^{0}\left(V_{i}\right)$ generates $Z_{O\left(V_{i}\right)}\left(V_{i}\right) / Z_{O\left(V_{i}\right)}^{0}\left(V_{i}\right)$, $i=1, \ldots, n_{1}$. We know each $V_{i}, i=1, \ldots, n_{1}$, has two isomorphism classes $V_{i}^{0}, V_{i}^{\delta}$ over $\mathbf{F}_{q}$. Let $m_{i}^{0}$ and $m_{i}^{\delta}$ be two elements in $\mathfrak{o}\left(V_{i}\right)\left(\mathbf{F}_{q}\right)$ corresponding to $V_{i}^{0}$ and $V_{i}^{\delta}$, respectively. We can assume $x=m_{1}^{0} \oplus m_{2}^{0} \oplus \cdots \oplus m_{n_{1}}^{0} \oplus m_{n_{1}+1}$. Let $\tilde{g}_{i}=$ $I d \oplus \cdots \oplus g_{i} \oplus \cdots \oplus I d, i=1, \ldots, n_{1}$. Then we have $\tilde{g}_{i} \in Z$ and $\tilde{g}_{i} \notin Z^{0}$, since $V_{1}^{0} \oplus \cdots \oplus V_{i}^{0} \oplus \cdots \oplus V_{n_{1}}^{0} \oplus V_{n_{1}+1} \not V_{1}^{0} \oplus \cdots \oplus V_{i}^{\delta} \oplus \cdots \oplus V_{n_{1}}^{0} \oplus V_{n_{1}+1}$ (Corollary 6.16). We also have that the images of $\tilde{g}_{i_{1}} \cdots \tilde{g}_{i_{p}}$ 's, $1 \leq i_{1}<\cdots<i_{p} \leq n_{1}, p=1, \ldots, n_{1}$, in $Z / Z^{0}$ are not equal to each other. Moreover, $\tilde{g}_{i}^{2} \in Z^{0}$. Thus the $\tilde{g}_{i} Z^{0}$ 's generate a subgroup $\left(\mathbb{Z}_{2}\right)^{n_{1}}$ in $Z / Z^{0}$.

(ii) Let us write $Z=Z_{S O(V)}(x)$ and $Z^{0}=Z_{S O(V)}^{0}(x)$ for simplicity. Assume $n_{2} \geq 1$. We know that the group $Z / Z^{0}$ has $2^{n_{2}-1}$ conjugacy classes since the $G$-orbit of $x$ splits into $2^{n_{2}-1} G\left(\mathbf{F}_{q}\right)$-orbits in $\mathfrak{g}\left(\mathbf{F}_{q}\right)$ (we can assume $q$ large enough). The same argument as in (i) shows that $\left|Z\left(\mathbf{F}_{q}\right)\right|=2^{n_{2}-1} q^{\operatorname{dim}(Z)}+$ lower terms. Then it follows that $Z / Z^{0}$ is an abelian group of order $2^{n_{2}-1}$. It is enough to show that there is a subgroup $\left(\mathbb{Z}_{2}\right)^{n_{2}-1} \subset Z / Z^{0}$. Let $1 \leq i_{1}, \ldots, i_{n_{2}} \leq s$ be such that $\chi\left(\lambda_{i_{j}}\right)>\lambda_{i_{j}} / 2$ and $\chi\left(\lambda_{i_{j}}\right)+\chi\left(\lambda_{i_{j}+1}\right) \leq \lambda_{i_{j}}, j=1, \ldots, n_{2}$.

Case 1: $\chi\left(\lambda_{s}\right)=\lambda_{s} / 2$. Then $i_{n_{2}}<s$. Let $V_{j}=\left(\lambda_{i_{j-1}+1}\right)_{\chi\left(\lambda_{i_{j-1}+1}\right)}^{m_{i_{j-1}+1}} \cdots\left(\lambda_{i_{j}}\right)_{\chi\left(\lambda_{i_{j}}\right)}^{m_{i_{j}}}$, $j=1, \ldots, n_{2}+1$, where $i_{0}=0, i_{n_{2}+1}=s$. Then $V=V_{1} \oplus \cdots \oplus V_{n_{2}+1}$. We have $Z_{O\left(V_{i}\right)}\left(V_{i}\right) / Z_{O\left(V_{i}\right)}^{0}\left(V_{i}\right)=\mathbb{Z}_{2}, i=1, \ldots, n_{2}$ and $Z_{O\left(V_{n_{2}+1}\right)}\left(V_{n_{2}+1}\right) / Z_{O\left(V_{n_{2}+1}\right)}^{0}\left(V_{n_{2}+1}\right)$ $=\{1\}$. Take $g_{i} \in Z_{O\left(V_{i}\right)}\left(V_{i}\right)$ such that $g_{i} Z_{O\left(V_{i}\right)}^{0}\left(V_{i}\right)$ generates $Z_{O\left(V_{i}\right)}\left(V_{i}\right) / Z_{O\left(V_{i}\right)}^{0}\left(V_{i}\right)$, $i=1, \ldots, n_{2}$. Let $\tilde{g}_{i}=g_{1} \oplus I d \oplus \cdots \oplus g_{i} \oplus \cdots \oplus I d, i=2, \ldots, n_{2}$. We have $\tilde{g}_{i} \in Z$ and $\tilde{g}_{i} \notin Z^{0}$. We also have the images of $\tilde{g}_{i_{1}} \cdots \tilde{g}_{i_{p}}$ 's, $2 \leq i_{1}<\cdots<i_{p} \leq n_{2}$, $p=1, \ldots, n_{2}-1$, in $Z / Z^{0}$ are not equal to each other. Moreover, $\tilde{g}_{i}^{2} \in Z^{0}$. Hence the $\tilde{g}_{i} Z^{0}$ s generate a subgroup $\left(\mathbb{Z}_{2}\right)^{n_{2}-1}$ in $Z / Z^{0}$.

Case 2: $\chi\left(\lambda_{s}\right)>\lambda_{s} / 2$. Then $i_{n_{2}}=s$. Let $V_{j}=\left(\lambda_{i_{j-1}+1}\right)_{\chi\left(\lambda_{i_{j-1}+1}\right)}^{m_{i_{j-1}+1}} \cdots\left(\lambda_{i_{j}}\right)_{\chi\left(\lambda_{i_{j}}\right)}^{m_{i_{j}}}$, $j=1, \ldots, n_{2}$, where $i_{0}=0$. Then $V=V_{1} \oplus \cdots \oplus V_{n_{2}}$. We have $Z_{O\left(V_{i}\right)}\left(V_{i}\right) / Z_{O\left(V_{i}\right)}^{0}\left(V_{i}\right)$ $=\mathbb{Z}_{2}, i=1, \ldots, n_{2}$. Take $g_{i} \in Z_{O\left(V_{i}\right)}\left(V_{i}\right)$ such that $g_{i} Z_{O\left(V_{i}\right)}^{0}\left(V_{i}\right)$ generates $Z_{O\left(V_{i}\right)}\left(V_{i}\right) / Z_{O\left(V_{i}\right)}^{0}\left(V_{i}\right), i=1, \ldots, n_{2} . \quad$ Let $\tilde{g}_{i}=g_{1} \oplus I d \oplus \cdots \oplus g_{i} \oplus \cdots \oplus I d$, $i=2, \cdots, n_{2}$. The $\tilde{g}_{i} Z^{0}$ 's generate a subgroup $\left(\mathbb{Z}_{2}\right)^{n_{2}-1}$ in $Z / Z^{0}$.

\section{Complements}

In this section we give an example in type $B_{4}$. We also make some comments on the explicit Springer correspondence.

8.1. Example. We list the unipotent classes in $S O(9)$ and nilpotent classes in $\mathfrak{s o}(9)$. We use the notation in 2]; see also Section 1.

Unipotent classes in characteristic 2 :

$\underline{8_{5} 1_{1}}, \underline{6_{4} 2_{2} 1_{1}}, \underline{6_{4} 1_{1}^{3}}, \underline{4_{3}^{2} 1_{1}}, 4_{2}^{2} 1_{1}, 4_{3} 2_{2}^{2} 1_{1}, \underline{4_{3} 2_{1}^{2} 1_{1}}, 4_{3} 2_{2} 1_{1}^{3}, \underline{4_{3} 1_{1}^{5}}, 3_{2}^{2} 2_{2} 1_{1}, \underline{3_{2}^{2} 1_{1}^{3}}$, $2_{2}^{4} 1_{1}, 2_{1}^{4} 1_{1}, 2_{2}^{3} 1_{1}^{3}, 2_{2}^{2} 1_{1}^{5}, 2_{1}^{2} 1_{1}^{5}, 2_{2} 1_{1}^{7}, 1_{1}^{9}$.

Nilpotent classes in characteristic 2:

$$
\begin{aligned}
& 5_{5} 4_{4}, 4_{4}^{2} 1_{1}, \underline{4_{3}^{2} 1_{1}}, 4_{2}^{2} 1_{1}, 4_{4} 3_{3} 1_{1}^{2}, 3_{3}^{2} 1_{1}^{3}, \underline{3_{2}^{2} 1_{1}^{3},} 3_{3}^{2} 2_{2} 1_{1}, 3_{2}^{2} 2_{2} 1_{1}, 3_{3} 2_{2}^{3}, 3_{3} 2_{2} 1_{1}^{4}, \\
& 2_{2}^{4} 1_{1}, 2_{1}^{4} 1_{1}, 2_{2}^{3} 1_{1}^{3}, 21_{2}^{2} 1_{1}^{5}, 2_{1}^{2} 1_{1}^{5}, 2_{2} 1_{1}^{7}, 1_{1}^{9} .
\end{aligned}
$$


Unipotent/nilpotent classes in characteristic not 2:

$$
9_{5}, \underline{7_{4}^{1} 1_{1}^{2}}, \underline{\underline{5_{3}^{1} 3_{2}^{1} 1_{1}^{1}}}, 5_{3}^{1} 2_{1}^{2}, \underline{5_{3}^{1} 1_{1}^{4}}, 4_{2}^{2} 1_{1}^{1}, 3_{2}^{3}, \underline{3_{2}^{2} 1_{1}^{3}}, \underline{3_{2}^{1} 2_{1}^{2} 1_{1}^{2}}, \underline{3_{2}^{1} 1_{1}^{6}}, 2_{1}^{4} 1_{1}^{1}, 2_{1}^{2} 1_{1}^{5}, 1_{1}^{9} .
$$

In each case the component group of the centralizer is trivial except that the component groups for the underlined ones are $\mathbb{Z} / 2 \mathbb{Z}$ and for the double underlined one is $(\mathbb{Z} / 2 \mathbb{Z})^{2}$. In this case the number of unipotent classes and nilpotent classes over an algebraically closed field of characteristic 2 happen to be the same, but this is not true in higher ranks. Note that the component groups are already quite different.

8.2. In [9], Spaltenstein shows that the explicit Springer correspondence for the pairs $\left(\mathrm{c}, \overrightarrow{\mathbb{Q}}_{l}\right)$ is given by the pairs of partitions associated by him to each orbit, assuming the theory of Springer representation is valid. We will show elsewhere that the explicit correspondence for the pairs $(c, \mathcal{F})(\mathcal{F}$ nontrivial $)$ is given by the pairs of partitions appearing in the proof of Proposition 5.1

The explicit generalized Springer correspondence for the unipotent case in characteristic 2 is described by Lusztig and Spaltenstein in 8 for classical groups. The correspondence is more complicated than the nilpotent case because of the existence of cuspidal local systems. In the unipotent case various Weyl groups for Levi subgroups are needed and in our case we only need the Weyl group for $G$.

\section{ACKNOWLEDGEMENT}

I would like to thank Professor George Lusztig for his guidance, encouragement and help during this research. I am also grateful to the referee for the valuable suggestions.

\section{REFERENCES}

[1] A. Beilinson, J. Bernstein and P. Deligne, Faisceaux pervers. Asterisque 100(1981). MR.751966 (86g:32015)

[2] W.H. Hesselink, Nilpotency in classical groups over a field of characteristic 2. Math. Z. 166(1979), 165-181. MR525621 (82d:14030)

[3] S. Kato, On the geometry of exotic nilpotent cones. (2006) arxiv: 0607478v1.

[4] G. Lusztig, Intersection cohomology complexes on a reductive group. Invent. Math. 75(1984), no. 2, 205-272. MR732546 (86d:20050)

[5] G. Lusztig, Character sheaves on disconnected groups. II. Represent. Theory 8(2004), 72-124 (electronic). MR2048588 (2006d:20090b)

[6] G. Lusztig, Character sheaves II. Adv. in Math. 57(1985), no. 3, 226-265. MR806210 $(87 \mathrm{~m}: 20118 \mathrm{a})$

[7] G. Lusztig, A class of irreducible representations of a Weyl group. Nederl. Akad. Wetensch. Indag. Math. 41(1979), no. 3, 323-335. MR546372 (81a:20052)

[8] G. Lusztig and N. Spaltenstein, On the generalized Springer correspondence for classical groups. Algebraic groups and related topics (Kyoto/Nagoya, 1983), 289-316, Adv. Stud. Pure Math., 6, North-Holland, Amsterdam, 1985. MR803339 (87g:20072a)

[9] N. Spaltenstein, Nilpotent Classes and Sheets of Lie Algebras in Bad Characteristic. Math. Z. 181(1982), 31-48. MR671712 (83m:17007)

[10] N. Spaltenstein, Classes unipotentes et sous-groupes de Borel. Lecture Notes in Mathematics, 946. Springer-Verlag, Berlin-New York, 1982. MR672610 (84a:14024)

[11] N. Spaltenstein, Nilpotent classes in Lie algebras of type $F_{4}$ over fields of characteristic 2. J. Fac. Sci. Univ. Tokyo Sect. IA Math. 30(1984), no. 3, 517-524. MR731515 (85g:20056)

[12] T.A. Springer, Trigonometric sums, Green functions of finite groups and representations of Weyl groups. Invent. Math. 36(1976), 173-207. MR0442103 (56:491)

[13] T.A. Springer, Linear algebraic groups. Second edition. Progress in Mathematics, 9. Birkhäuser Boston, Inc., Boston, MA, 1998. MR.1642713 (99h:20075) 
[14] R. Steinberg, Conjugacy classes in algebraic groups. Lecture Notes in Mathematics, Vol. 366. Springer-Verlag, Berlin-New York, 1974. MR0352279 (50:4766)

[15] R. Steinberg, On the desingularization of the unipotent variety. Invent. Math. 36 (1976), 209-224. MR0430094 (55:3101)

[16] T. Xue, Nilpotent orbits in classical Lie algebras over $F_{2^{n}}$ and the Springer correspondence. Proc. Natl. Acad. Sci. USA 105 (2008), no. 4, 1126-1128. MR2375447(2009c:14096)

Department of Mathematics, Massachusetts Institute of Technology, Cambridge, MassachusetTs 02139

E-mail address: txue@math.mit.edu 